OECDpublishing

\title{
ASSESSING NATIONAL ACTION PLANS ON ANTIMICROBIAL RESISTANCE IN ANIMAL PRODUCTION:
}

WHAT LESSONS CAN BE DRAWN?

OECD FOOD, AGRICULTURE AND FISHERIES

PAPER

March $2021 \mathbf{n}^{\circ} \mathbf{1 5 3}$ 


\title{
OECD TRADE AND AGRICULTURE DIRECTORATE
}

\section{Assessing National Action Plans on Antimicrobial Resistance in Animal Production: What Lessons Can be Drawn?}

\author{
Michael Ryan
}

\begin{abstract}
Global efforts to combat the risks posed by antimicrobial resistance (AMR) depend on effective implementation of national action plans. Most countries have taken a One Health approach in developing their national action plans covering human health, animal health and the environment. This study assesses the evidence on the implementation of these plans in livestock agriculture across six OECD countries, as well as in Brazil, the People's Republic of China, and the Russian Federation. The findings highlight the need for greater co-ordination and coherence amongst stakeholders in implementing workable solutions, including developing integrated surveillance and monitoring systems, improving regulations on the availability of antibiotics, and improving on-farm biosecurity practices. Additional efforts to enhance public awareness, to improve education and training, and to explore alternative interventions and innovations to antimicrobials in animal health and animal welfare could improve outcomes in this area. The lack of longterm funding continues to hinder the implementation of national action plans on AMR.
\end{abstract}

Key words: One Health, biosecurity, disease prevention, critically important antibiotics

JEL codes: Q13

\section{Acknowledgements}

The author wishes to acknowledge the valuable feedback provided by OECD Delegations and the members of the Expert Steering Group on AMR, who helped to improve the clarity and quality of this report. Special thanks are also extended to Lee Ann Jackson, Michele Cecchini, Jonathan Brooks, and Julia Neilson for their constructive comments and suggestions throughout the review process.

A previous version of this paper was released on 30 March 2021. This version updates the section on Canada (pp. 14-17). 


\section{Table of contents}

$\begin{array}{ll}\text { Abbreviations and acronyms } & 3\end{array}$

$\begin{array}{ll}\text { Glossary } & 5\end{array}$

$\begin{array}{ll}\text { 1. Background } & 8\end{array}$

2. Objectives and scope $\quad 9$

3. Analytical approach and data sources 10

4. Implementation of the National Action Plans on AMR in animal agriculture 11

$\begin{array}{lr}\text { Australia } & 12\end{array}$

Canada $r$

$\begin{array}{lr}\text { Denmark } & 17\end{array}$

$\begin{array}{lr}\text { Ireland } & 20\end{array}$

$\begin{array}{lr}\text { Japan } & 22\end{array}$

$\begin{array}{lr}\text { United States } & 25\end{array}$

\begin{tabular}{lr} 
Brazil & 27 \\
\hline
\end{tabular}

$\begin{array}{lr}\text { China } & 30\end{array}$

$\begin{array}{lr}\text { Russian Federation } & 32\end{array}$

5. Lessons learned and best practices to contain the risks of AMR 34

Policies to prevent disease outbreaks are central to addressing the risks of AMR 36

Good regulations that encourage the responsible use of antibiotics in food animal production 36

Integrated and reliable surveillance and monitoring systems of antimicrobial use and

$\begin{array}{ll}\text { antimicrobial resistance } & 37\end{array}$

Well-functioning and coherent administrative structures to include all stakeholders 37

Having adequate funding to implement the national action plans $\quad 37$

Outstanding challenges and shortcomings of the national action plans 38

$\begin{array}{ll}\text { References } & 39\end{array}$ 


\section{Abbreviations and acronyms}

\begin{tabular}{|c|c|}
\hline AGAR & Australian Group on Antimicrobial Resistance \\
\hline AMRPC & Antimicrobial Resistance Prevention and Containment Steering Group \\
\hline AMRSC & Antimicrobial Resistance Standing Committee \\
\hline$A B C$ & Antibiotic consumption \\
\hline AGP & Antimicrobial growth promoters \\
\hline AMR & Antimicrobial resistance \\
\hline AMS & Antimicrobial stewardship \\
\hline AMU & Antimicrobial use \\
\hline ASP & Antimicrobial Stewardship Programme \\
\hline BRIICS & Brazil, Russian Federation, India, Indonesia, China and South Africa \\
\hline $\mathrm{CAHI}$ & Canadian Animal Health Institute \\
\hline CARSS & Canadian Antimicrobial Resistance Surveillance System \\
\hline CBA & Cost-benefit analysis \\
\hline ClAs & Critically important antimicrobials \\
\hline CVMA & Canadian Veterinary Medical Association \\
\hline EARS-Net & European Antimicrobial Resistance Surveillance Network \\
\hline ECDC & European Centre for Disease Prevention and Control \\
\hline EFSA & European Food Safety Authority \\
\hline ELS & Education, Labour and Social Affairs Directorate \\
\hline EMA & European Medicines Agency \\
\hline ESAC-Net & European Surveillance of Antimicrobial Consumption Network \\
\hline ESG & Expert Steering Group \\
\hline ESVAC & European Surveillance of Veterinary Antimicrobial Consumption \\
\hline EU & European Union \\
\hline FAO & Food and Agriculture Organisation of the United Nations \\
\hline HPCIA & Highest Priority Critically Important Antimicrobials \\
\hline HPRA & Health Products Regulatory Authority \\
\hline IOs & International Organisations \\
\hline JIACRA & Joint Interagency Antimicrobial Consumption and Resistance Analysis \\
\hline JPIAMR & Joint Programming Initiative on Antimicrobial Resistance \\
\hline LMIC & Low and Middle Income Countries \\
\hline MDRO & Multi-Drug Resistant Organism \\
\hline MIA & Medically Important Antimicrobials \\
\hline MRSA & Methicillin-resistant Staphylococcus aureus \\
\hline NAP & National Action Plan \\
\hline
\end{tabular}


NGO

OECD

OIE

PCU

PHAC

R\&D

SDGs

USDA

WB

WHO

WTO
Non-governmental organisation

Organisation for Economic Cooperation and Development

World Organisation for Animal Health

Population Correction Unit

Public Health Agency of Canada

Research and Development

Sustainable Development Goals

United States Department of Agriculture

World Bank

World Health Organization

World Trade Organization 


\section{Glossary}

Antibiotic

Antimicrobials

Acquired resistance

Companion animals

Epidemiology

Infection

Ionophores

Metaphylaxis

Multidrug resistant

One Health approach

Pathogens

Prophylaxis

Therapeutic

Medically Important Antimicrobials (MIA)

Critically Important Antimicrobials (CIA)
An antimicrobial agent that kills or slows the growth of bacteria.

Human and animal medicines that are used to treat infections.

Occurs through mutation in bacterial DNA or by getting the resistance genes through horizontal gene transfer when DNA moves from one bacterium to another.

Domestic pets such as cats, dogs, and sport animals.

The study of the spread of disease.

The entry and multiplication of an infectious agent in animals or humans.

Compounds that facilitate the transmission of an ion across a lipid barrier by combining with the ion or by increasing the permeability of the barrier.

Treatment of a group of animals to prevent the spread of a disease

A bacterial pathogen that is resistant to two or more antimicrobial classes

An approach to address a health threat at the human-animal-environment interface based on collaboration, communication, and co-ordination across all relevant sectors and disciplines, with the goal of achieving optimal health outcomes for people and animals. It is applicable at the subnational, national, regional, and global level.

Bacteria, viruses, parasites and fungi that can cause disease.

Preventative treatment of animals against the outbreak of a disease.

Treatment of animals against a specific disease.

Antimicrobial classes used in human medicine and listed on the WHO-CIA List.

Antimicrobials that provide the sole therapy to treat serious bacterial infections in humans. 


\section{Key messages}

\section{What are the issues and why are they important?}

- Antimicrobial resistance (AMR) poses a major threat to human health, animal and plant health, and environmental health. Due to the global nature of AMR, the emergence of resistance can have potentially huge economic and social costs in all countries.

- Having recognised this threat, in 2015 the World Health Organization, in collaboration with the Food and Agriculture Organization of the United Nations (FAO) and the World Organisation for Animal Health (OIE), developed a Global Action Plan (GAP) to contain the rise in antimicrobial resistance (AMR) in humans and animals. The GAP provided a useful starting point to guide Member countries as they developed their country-specific National Action Plans (NAP) to mitigate the impacts of antimicrobial resistance. Many countries have developed NAPs to combat the risks of AMR under the One Health framework.

\section{What did we learn?}

- Most countries have adopted a mix of policies to tackle AMR by influencing the behaviour and husbandry practices of livestock producers. There is no single measure or solution to tackling the challenges posed by AMR in animal agriculture, but a mix of good policy measures, regulations, good husbandry practices, and biosecurity measures can successfully and sustainably reduce the need for antibiotics in livestock production and consequently lower the risks of AMR.

- The starting point is different in every country due to the different farming systems, different economic incentives, policy/regulatory measures, and different technical capacities. OECD countries typically have well-functioning surveillance and monitoring systems and veterinary capacity, whereas this infrastructure is generally weak in developing countries.

- Good farm management and husbandry practices are critical to tackling AMR in animals. In many countries, the selective use of vaccines under veterinary supervision has proven to be effective in reducing the outbreaks of infectious diseases. The adoption of biosecurity measures both on and off the farm can limit the need for antibiotics by controlling the introduction of pathogens.

- In many countries, lack of funding has impeded the implementation of measures to combat AMR. Much of the public funding is allocated to dealing with AMR in human health, and limited funding is available to implement measures to tackle AMR in animal health. However, new initiatives have emerged involving collaboration and partnerships between the public and private sector. In this context, it is critical that countries have the political willpower and determination to ensure the effective implementation of the measures in their NAPs to combat AMR.

- National regulations play an important role in controlling the availability and use of antimicrobials in animal production. Strict controls on the sale of antibiotics for food animals through the implementation of veterinary prescriptions for antibiotics helps to limit over-use of antibiotics in animal production. This is particularly important to ensuring that the High Priority-Critically Important Antibiotics are not used inappropriately in animal agriculture. While good regulations on antibiotics are important to ensure the proper treatment of animals, veterinarians also play a central role by prescribing the appropriate interventions to ensure good animal health and good animal welfare.

- At the farm level, antimicrobial use (AMU) data allows for accurately measuring AMU, and enables the provision of targeted advice and guidance. Reliable and accurate data on the use of antibiotics by different animal species, and at different stages of production, is not yet available in any country. 


\section{Key recommendations}

- Successful AMR mitigation strategies include preventative measures that limit and prevent infectious disease outbreaks in food animals. Greater attention also needs to be given to the development of alternative interventions to antibiotics in the prevention and treatment of animal diseases, in particular, a greater focus on enhancing animal husbandry, nutrition, and biosecurity measures on livestock farms.

- Regulations that encourage the responsible use of antibiotics in food animal production require robust enforcement. Further efforts are needed to improve regulations and enforcement on the appropriate use of antimicrobials in human and animal health.

- A standardised approach to data collection by the national surveillance systems would allow countries to establish "benchmarking" for antibiotic usage in the different animal sectors. The adoption of international standards for the collection and reporting on antibiotic use and antibiotic resistance in animals would help support comparative analysis within commodities to mitigate AMR across countries.

- Additional efforts are needed to ensure transparency and coherence in the implementation of national policies to combat AMR. Sharing of experiences across different administrative structures and institutions in different sectors and countries would help improve the co-operation among public policy institutions and the private stakeholders.

- In order to enhance public confidence and motivation, governments should commit to the longterm funding required to implement national actions plans. Finding adequate funding is a major challenge for most countries. 


\section{Background}

In May 2015, the World Health Assembly acknowledged the threat to human and animal health arising from the development and spread of antimicrobial resistant bacteria. In response to this threat, the World Health Organisation (WHO) developed the Global Action Plan (GAP) in collaboration with the FAO and OIE (and endorsed by all Member countries), to combat the rise in antimicrobial resistance (WHO, 2015). This Plan sets out a broad framework with specific objectives to tackle antimicrobial resistance (AMR). These include improving awareness and understanding of AMR; strengthening knowledge through surveillance and research; reducing the incidence of infection; optimizing the use of antimicrobials; and, developing the economic case for sustainable investment to meet the specific needs of all countries, as well as greater investment in new medicines, diagnostic tools, vaccines and other interventions. In essence, the GAP adopts the One Health approach and involves close co-operation amongst different stakeholders across human medicine, veterinary medicine, animal and plant production, environment and consumers. In 2017, the World Health Assembly advised its Member countries to develop National Actions Plans (NAPs) on antimicrobial resistance to combat the risks posed by AMR.

The development of NAPs is an essential first step to containing antimicrobial resistance. These plans enable a more co-ordinated, coherent, and cross sectoral approach to mitigating the impacts of AMR in human and animal health. In practice, the GAP has provided a framework to guide countries as they develop their specific NAPs to combat antimicrobial resistance in human and animal health. The GAP outlines several strategic objectives as well as possible actions to contain the rise in resistant pathogens over a five to tenyear period (WHO, 2015). As part of the One Health approach, most countries have developed national plans that are tailored to their specific circumstances with respect to human health, animal health and welfare and environmental health. To-date more than four-fifths of WHO Member countries have developed national action strategies and plans for dealing with AMR, of which, more than half of these countries have developed these based on the One Health approach covering, human, animal and environmental health (Binder, 2019). More than half of the low and middle income countries have national measures in place to deal with AMR in human health, but less than $10 \%$ have national AMR surveillance systems in place for food producing animals. In some OECD countries, efforts to tackle the emergence and spread of AMR have been in place for several decades, and many of these countries have developed robust surveillance systems on antimicrobial use and on the prevalence of antimicrobial resistance.

In most countries, the NAPs have brought together ongoing work on AMR from the different sectors under the One Health framework, and this facilitates an economy-wide, coherent response to the AMR challenge. While tackling AMR is a priority in most countries, the implementation of the action plans and mitigation measures varies substantially by country. In many countries, the implementation of actions on AMR in human health is more advanced than in the animal, plant and environmental sectors, and this may be due to the additional complexities associated with monitoring antimicrobial use in animal production and the lack of reliable information and data at the farm level.

This project complements the ongoing work on antimicrobial use and antimicrobial resistance in several international organisations including the World Organisation for Animal Health (OIE), the Food and Agriculture Organization of the United Nations (FAO), and the WHO. In 2017, the UN Secretary General and the WHO Director General established the Inter-Agency Co-ordination Group (IACG) on antimicrobial resistance. The core mandate of the IACG was to provide guidance on sustainable approaches to effective global action to address the complex challenge posed by AMR. A final report with recommendations was presented to the UN Secretary General in mid-2019. As part of this effort, the OIE (2015) has been collecting data on antimicrobial use in animals based on the related OIE standards and in line with the Global Action Plan. The WHO, OIE and the FAO have developed a tripartite questionnaire to track progress in the implementation of national action plans, based on countries self-assessment of their progress in developing national antimicrobial resistance action plans and their success in the implementation of these actions to address AMR. In 2019, the Tripartite established the AMR Multi-Partner Trust Fund (AMR-MPTF) to support countries efforts to counter the threat of AMR through the development and implementation of their NAPs on AMR.

This paper is organised in five sections, as follows. Section 2 outlines the objectives and scope of the work. Section 3 describes the approach taken to assessing the national action plans on AMR in animal health. Section 4 provides a summary of the national action plans in six OECD countries - Australia, Canada, 
Denmark, Ireland, Japan, and the United States, as well as in Brazil, the People's Republic of China (hereafter "China") and the Russian Federation (hereafter "Russia"). Section 5 summarises the lessons learned and best practices to optimize the use of antimicrobials in animal production and to contain the rise in antimicrobial resistance.

\section{Objectives and scope}

The current work assesses national strategies and plans to combat antimicrobial resistance in livestock to assist policy makers in choosing the appropriate mix of polices to contain AMR in food producing animals. ${ }^{1}$ The objectives of this study are to identify the policies and best practices that have been successful in optimizing the use of antibiotics in animal production, and, thus reducing the selection pressure on microorganisms to develop resistance. ${ }^{2}$ While member countries of the WHO have adopted the broad framework as set out in the GAP, countries have taken different approaches to implementing their national action plans on AMR in animal agriculture. This assessment provides a comparative analysis of the different approaches countries have taken to tackle AMR in food producing animals. The work assesses the success of the policies and measures as outlined in the national action plans in containing the risks of AMR in animal agriculture in six OECD countries (Australia, Canada, Denmark, Ireland, Japan and the United States), Brazil, China, and Russia. The findings of this assessment are aimed at helping policy makers to further refine and enhance their NAPs, based on the lessons learned and best practices of the selected countries in tackling AMR in food producing animals.

While this study is based on a qualitative analysis, a quantitative analysis using the One Health model on the impacts of AMR on human health and animal health is being developed in the Directorate for Employment, Labour and Social Affairs (ELS). This modelling work on AMU/AMR in livestock agriculture draws on the ongoing work of the OECD Committee for Agriculture, as well as input from the Expert Steering Group on AMR in animal agriculture. One of the key challenges is how to persuade animal producers to change their behaviour and to adopt alternative disease prevention measures in animal production, while maintaining good animal health and welfare and raising farm incomes. In many situations, farmers' behaviour is related to their experience of the technical and cost effectiveness of antibiotics to control animal diseases and maintain good animal health and productivity on the farm.

The aim of this study is to identify the best practices and policies for containing AMR by drawing on the experience of a number of OECD and non-OECD countries. The findings of this work will provide policy makers a better understanding of the complexities of AMU and AMR and the mix of practices required to contain AMR in different contexts. Moreover, the findings may shed light on the administrative complexities at national level in implementing national action plans based on the One Health approach to tackling AMR. Policies to combat AMR and farming practices to prevent disease outbreaks in animal production are challenging and vary by country, region, as well as by the species of animal and the type of production system. Lastly, AMR can impact human health, food security, food safety, and farm viability, as well as animal, plant and environmental health.

Countries have clearly stated goals and objectives outlined in their NAPs on the use of antibiotics in human and in animal health, and these goals reflect the economic incentives, technical capacities and environmental commitments, as well as the animal production system in the country. Identifying the appropriate mix of solutions in tackling AMR in animal production is complex and relates to the technical efficiency of the antibiotics, as well as the type of farming system and the availability of alternative treatments at the farm level. However, all actions aimed at tackling the risks of AMR contribute to a broader public goods benefit in terms of better human, animal and environmental health, and can lead to more sustainable food animal production in the long run.

\footnotetext{
${ }^{1}$ This work follows on from the recently published "Evaluating the Economic Benefits and Costs of Antimicrobial Use in Food Producing Animals" (OECD, 2019).

${ }^{2}$ Companion animals, agricultural crops, and aquaculture are not covered here.
} 
The terms "medically important antimicrobials" (MIA) and "critically important antimicrobials" (CIA) as used in this paper follow the definitions set out by the WHO (2017). More specifically, MIA are the antimicrobial classes used in human medicine and listed on the WHO-CIA List where they are categorized according to specified criteria; as "important", "highly important" or "critically important" for human medicine. The MIA also includes antimicrobials used in non-medical settings (e.g. food producing animals) that are included in the same class as those used in human medicine, and where there is the potential for these antimicrobials to select for resistance to human pathogens. For example, the veterinary antimicrobial enrofloxacin, and the medical drug ciprofloxacin belong to the fluoroquinolones class of antimicrobials. Critically important antimicrobials are defined as the classes of antimicrobials that provide the sole therapy, or one of limited available therapies, to treat serious bacterial infections in humans. In addition, the OIE publishes a list of critically important antimicrobials for animal health and this list is reviewed annually by Member countries. Also, countries may have their own national lists of critically important antimicrobials based on national risks.

\section{Analytical approach and data sources}

The National Action Plans for tackling AMR focus on the cross-sectoral actions and behavioural changes that have an impact on human health, animal and plant health, and the environment. Given this complexity, a conventional economic assessment based on a single intervention would not be appropriate. Since a mix of policies and practices are required to deal with AMR, any analysis requires both a qualitative and quantitative assessment of the mitigation measures. This study undertakes a qualitative assessment of the NAPs for six OECD countries and three non-OECD countries, and examines the technical aspects, and especially the use of highest priority critically important antibiotics in food producing animals.

Specifically, this study assesses the changes in antimicrobial use on animal farms following the implementation of the NAPs in the selected countries, and examines the impact of these changes on the overall objectives and targets as set out in the NAPs.

Changes in the production behaviour of producers in terms of their use of antibiotic in production is assessed at the sector level and, where data is available, at the species level. The baseline information available set out in the strategic plans is taken as the reference point from which progress is assessed. While many countries have phased out the use of antibiotics for growth promotion, changes in antibiotics usage in these countries largely relate to reducing the over-use of antibiotics in disease prophylaxis and to improving disease diagnosis for therapeutic purposes.

This analysis covers the major food producing animals; pigs, poultry, and beef and dairy cows for six OECD countries, as well as Brazil, China and Russia. The Secretariat has been working closely with OECD and non-member countries with the view to getting good regional representation, subject to the availability of robust and reliable data on AMU and AMR in animal production. The heterogeneity of animal production between different countries and regions increases the complexity of assessing the national strategies and the cost effectiveness of different disease preventative measures in animal production.

The six OECD countries provided information and data to the Secretariat on antimicrobial use and antimicrobial resistance in animal production. These countries reflect the diversity in approaches and policies taken to addressing the use of antibiotics in food producing animals. This assessment uses data primarily from national sources, but also from several international organisations that are involved in the monitoring of AMR. For example, data on the veterinary use of antibiotics in farm animals is also drawn from the annual publications of the European Medicines Agency (EMA), the OIE Annual Reports on Antimicrobial Agents intended for use in animals, and FAO reports.

To enable cross-country comparisons of the NAPs it is important to have standardised measures or indicators of antibiotic use and antibiotic resistance. These indicators should ideally cover changes in the overall use of antibiotics by sector, as well as by class of antibiotics, with particular attention to last resort antibiotics. Subject to the availability of information, this study also attempts to assess the economic costs associated with the development and implementation of the strategic plans on AMR in animal production. However, in many countries the costs of implementing their NAPs are included in the country's annual animal disease control and eradication programme budgets and are difficult to decipher. 
The overarching global goal of optimizing the use of antibiotics in animal production, while maintaining animal productivity, good animal health and welfare, is a formidable challenge for all countries. While identifying the best practices and policies is an important first step in the process of limiting the risks of AMR, a second step could involve an in-depth analysis of the cost-effectiveness or mitigation costs of alternative interventions and practices to antibiotics in animal production.

\section{Implementation of the National Action Plans on AMR in animal agriculture}

Member countries of the WHO, FAO and OIE have committed to the global efforts to combat the risk of AMR, under the framework of the GAP on AMR. The main goals of the GAP are to ensure the continuity of successful antimicrobial treatments, the prevention of infectious diseases, the effective use of antimicrobials that are quality assured, and the responsible use and access to needed antimicrobials (WHO, 2015). Most countries have developed their country specific national strategies and measures, as a first step to tackling the risks posed by AMR in humans, animals, and the environment. In order to meet the goals of the GAP, the Tripartite (WHO/FAO/OIE) developed a framework that identified technical, communication and educational measures under the following five strategic objectives.

- Improve awareness and understanding of antimicrobial resistance.

- Strengthen knowledge through surveillance and research.

- Reduce the incidence of infection.

- Optimize the use of antimicrobial agents.

- Develop the economic case for sustainable investment that takes account of the needs of countries, and increase investment in new medicines, diagnostic tools, vaccines, and other interventions.

There is currently limited information on the performance of the National Actions Plans reducing the risks of AMR in animal production, while maintaining good animal health, animal welfare and productivity. Moreover, limited consideration is given to the economic and social aspects of implementing a range of measures and policies to contain the risks of AMR in food producing animals.

In the European Union, the strategies on tackling AMR goes beyond the framework set out in the GAP. More specifically, the European Council has stated that Member States must set up specific national goals to manage antibiotic resistance and consumption in both human medicine and in veterinary to strengthen the One Health co-operation within the European Union. All EU Member States must set up specific national goals to manage antibiotic resistance and antibiotic use in both human medicine and veterinary medicine. In June 2017, the European Commission (EC) launched "A European One Health Action Plan" against antimicrobial resistance. This Action Plan supports EU Member States in establishing and monitoring their national action plans and brings together EU funds and institutions to promote innovation and research in this area.

The EU Action Plan emphasizes antibiotic stewardship, to optimize the use and extend the effective life of existing antibiotics. Recent legislation on veterinary medicines (EU) 2019/6 requires all Member States to collect farm level data on antibiotic use for pigs and poultry (from 2022) and other food producing species from 2027. Two EU Member States - Ireland and Denmark - have been selected for assessment in this study as they represent different, approaches to tackling AMR in food animal production.

This section summarises the NAPs in six OECD countries (Australia, Canada, Denmark, Ireland, Japan, and the United States), Brazil, China and Russia, and examines the preliminary findings of the measures to contain the rise in AMR. The lessons learned in this assessment are then discussed in terms of their potential to be generalised across other countries, regions and farming systems. 


\section{Australia}

Australia has taken a conservative approach in permitting the use of antibiotic in animal production. This has resulted in a relatively low level of antibiotic use. The use of high priority critically important antibiotics such as colistin, fourth generation cephalosporins and fluoroquinolones have either not been registered or approved for use in food producing animals. A 2014 report on antibiotic use and resistance in livestock set out recommendations for the surveillance and reporting of antimicrobial use and resistance in food producing animals.

The Australian Pesticides and Veterinary Medicines Authority (APVMA) is responsible for registering antimicrobial drugs for use in all animals, and for assessing the risks of AMR. Control over the supply of antimicrobials is stringent with nearly all antimicrobials used in animals subject to prescription by a veterinarian. In each state and territory, "a Veterinary Surgeon's Board" is responsible for controlling their supply, prescription and use. The 2017 APVMA report on resistance in animals examined the major AMR related issues associated with antimicrobial use in livestock. The report concluded that there is a low risk of AMR in livestock, and a low level of resistance to antibiotics that are currently used in human medicine.

\section{Australia's First National Antimicrobial Resistance Strategy 2015-2019 and Australia's National Antimicrobial Resistance Strategy 2020 and Beyond}

In June 2015, the Australian Department of Health, and then the Department of Agriculture, published Australia's First National Antimicrobial Resistance Strategy 2015-2019. This strategy took a cross-sectoral approach to the threat of antimicrobial resistance and focused primarily on bacterial resistance and the development of resistance to antibiotics. This strategy was developed with the participation of all stakeholders, including government and industry, and sets out actions and guidance for farmers, veterinarians, and health professionals to help reduce the emergence of resistant genes and bacteria.

The first strategy outlined seven objectives that require integrated and simultaneous actions, as progress in each of these areas is important to ensure a coherent and comprehensive response to the objectives of the strategy. The central goal of this strategy is to minimise the development and spread of AMR and ensure the continued availability of effective antimicrobials. The seven objectives are to:

- Increase awareness and understanding of AMR and its implications through effective communications, education and training.

- Implement effective antimicrobial stewardship practices across human health and animal health.

- Develop a nationally co-ordinated One Health surveillance of antimicrobial usage and AMR.

- Improve infection prevention and control measures across human health and animal health to prevent the spread of resistance.

- Develop a national research agenda on new products and approaches to prevent, detect and contain AMR.

- Strengthen international collaboration and partnerships to respond to AMR.

- Establish clear governance arrangements at the local, jurisdictional, national and international levels to ensure leadership, engagement and accountability to combat AMR.

The first strategy also sets out a framework to guide actions on antimicrobial use and resistance by encouraging co-ordination amongst stakeholders including, government, regulators, health care professionals, farmers, industry and researchers. Moreover, there are strict regulations on the prescription and use of antibiotics in animals, as well as comprehensive guidelines on the appropriate use of antibiotics and controls along the food chain.

The implementation and evaluation of the first strategy was supported by an Implementation Plan (2016), which set out specific actions, targets and timeframes, as well as specific indicators on the success of the first strategy. The Australian Antimicrobial Resistance Prevention and Containment (AMRPC) Steering Group (established in 2013), oversaw the implementation of this first strategy, and provided regular reports 
on the progress in combatting AMR. This Group is under the authority of the Department of Health and the Department of Agriculture, Water and the Environment.

Progress in implementing the first strategy is measured through the surveillance and data collection system that monitors changes in the usage of antibiotics, and the overall trend. A further goal is to establish benchmarking of antibiotic use by sector, which in turn, would allow for better targeting of policies to contain a rise in AMR.

Australia's National Antimicrobial Resistance Strategy 2020 and Beyond (2020 AMR Strategy) was endorsed on 13 March 2020. The 2020 AMR Strategy represents the expert views of stakeholders - from across governments, the animal and human health, and the environment, agricultural and food sectors - on how best to combat antimicrobial resistance. Implementation will be guided by a high-level One Health action plan, development of which has commenced, and by more detailed sector specific action plans.

\section{Findings}

In 2013, the Senate Finance and Public Administration References Committee's report "Progress in the Implementation of the Recommendations of the 1999 Joint Expert Technical Advisory Committee on Antibiotic Resistance (JETACAR) was released. The report concludes with ten recommendations to improve the monitoring and reporting of antibiotic usage and resistance in animals and humans, to strengthen antibiotic stewardship and infection control measures, and to increase investment in AMR research.

In Australia, all initiatives and actions to tackling AMR are underpinned by strong regulatory controls. For food producing animals, there are three levels of antibiotic controls. First, as all antibiotics are imported, there are strict custom controls at the point of entry. Second, all antimicrobials for use in animals must be assessed, registered and approved by APVMA. Third, in each state and territory the regulations on antibiotic use are strictly enforced. The process for registration of antibiotics takes into account whether antimicrobials destined for veterinary use are classified as being of high importance in human medicine under the Importance ratings and summary of antibacterial uses in humans and animal health in Australia (Australia's antibacterial ratings list). For example, fluoroquinolones are not approved for use in food-producing animals, although a number have been registered for use in companion animals.

An important outcome of the implementation of the JETACAR's recommendations has been the gradual removal of the remaining antibiotics for growth promotion from those classes considered important for human medicine, of which there were very few. Therefore, the only antibiotics now registered for growth promotion, which may also have other claims, in animals are limited to ionophores, flavophospholipol, olaquindox and avilamycin, which are not medically important for human health. ${ }^{3}$ Another important recommendation relates to the harmonisation of legislation on antibiotics across all States and Territories.

The 2014 APVMA report, "Quantity of Antimicrobial Products sold for Veterinary Use in Australia", gives a detailed breakdown of the sales of antimicrobial drugs over the period 2005 to 2010 . The overall volume of sales of veterinary antimicrobials at 644 tonnes showed little change over this period. Moreover, an average $98 \%$ of veterinary antimicrobial sales, from 2005 to 2010, was for food producing animals, with the remainder for non-food or companion animals. Of the antimicrobials sold, from 2005 to 2010 , for therapeutic purposes in food producing animals, poultry accounted for almost half $(49 \%)$ of total sales, and pigs accounted for about $36 \%$, while cattle and sheep accounted for the remaining $15 \%$. In terms of administration of the antibiotics in animals, over three-quarters of antimicrobials are given through the feed and about $18 \%$ through drinking water.

More specifically, the antimicrobial coccidiostats are mainly used in chickens and accounts for over half of all veterinary antibiotic sales (These drugs are not classified as antibiotic in the European Union). A relatively small amount of antimicrobials, estimated at $4-7 \%$, is used for growth promotion in food producing animals. However, an interesting trend has been an increase in sales of veterinary antimicrobials for companion animals, albeit from a low base.

\footnotetext{
${ }^{3}$ The role of ionophores in the development of AMR to important human drugs would be extremely low given the lack of ionophore use in human medicine and the evidence does not support the development of resistance. Also, lonophores are excluded from the OIE's AMU annual surveys.
} 


\section{Summary}

Australia has a comprehensive administrative system for the surveillance and monitoring of veterinary antimicrobial use and antimicrobial resistance in food producing animals. Each State or Territory has direct responsibility for regulating the use of antibiotics in animal production, and the implementation of measures to contain and lower antimicrobial resistance. The First National Antimicrobial Resistance Strategy 20152019 focussed on seven central objectives that include human health, animal health and welfare, agriculture, fisheries and the environment. The 2020 AMR Strategy continues the focus on these strategic objectives

The overall level of antibiotic use and antimicrobial resistance in food producing animals is low. In terms of therapeutic veterinary antibiotics use, almost $50 \%$ were sold to broiler chicken production as coccidiostats for the prevention of coccidiosis (a class of antimicrobials not used in humans and not considered to contribute to AMR risks in humans), and when combined with sales in the pig sector these account for over four-fifths of total annual antibiotic sales. While antibiotics not medically important for human health are still permitted for growth promotion in animal production, the use has been declining and now represents less than $8 \%$ of total sales. Australia has no growth promotion claims with currently registered antimicrobials medically important for human health. Australia's antibacterial ratings list provides a comprehensive summary of registered antibacterials used in animal health in the Australian context.

\section{Canada}

Antimicrobial resistance (AMR) is a priority for all levels of government in Canada (Federal, Provincial and Territorial), and multiple stakeholders. The responsibility for human and animal health, agriculture and agrifood is shared among the Federal, Provincial and Territorial governments. While the roles and responsibilities of each level of government in dealing with antimicrobial use (AMU) and AMR are distinct, they complement each other in tackling the challenges posed by AMR. The Provincial/Territorial jurisdictions are responsible for antimicrobial use practices, whereas the sales of antimicrobial drugs and other health products for both human and animal use are federally regulated under the Food and Drugs Act and its Regulations.

In 2014, Canada developed the Antimicrobial Resistance and Use in Canada: A Federal Framework for Action. This framework mapped out a co-ordinated federal approach to responding to the threat of AMR, and outlines the Government's key focus areas and plans for action. Building on the strategic areas of focus and priority actions outlined in the federal framework, Canada released the Federal Action Plan on Antimicrobial Resistance and Use in 2015, which identified concrete actions to be undertaken by the Federal Government. In 2018, a Progress Report on the Federal Action Plan, assessing the Federal Government's success in implementing its commitments was released. The government of Canada is working to prevent, limit, and control the emergence and spread of AMR by taking concrete actions in surveillance, stewardship, and innovation.

\section{Tackling Antimicrobial Resistance and Antimicrobial Use: A Pan-Canadian Framework for Action to address AMR}

In 2017, Canada published Tackling Antimicrobial Resistance and Antimicrobial Use: A Pan-Canadian Framework for Action. This Framework affirms the commitment of the Federal, Provincial and Territorial Governments to undertake co-ordinated actions on tackling AMR across the different sectors and jurisdictions. The overarching strategic objectives, as well as the specific actions are set out in the Framework under four pillars: surveillance, stewardship, infection prevention and control, and research and innovation. These strategic objectives include:

- Strengthen governance structures to generate knowledge and information on AMR and AMU in human, agriculture and animals through the monitoring, detection and tracking of resistant organisms to develop and monitor interventions.

- Promote, facilitate and measure appropriate AMU in humans and animals to conserve the effectiveness of antimicrobials that are critical to human and animal health, and to limit the development and spread of resistant organisms within and among populations. 
- Reduce the need for antimicrobial treatment by promoting infection prevention and control practices to decrease infection rates in healthcare, community and animal settings.

- Support the advancement of research and innovation approaches for the identification, characterization and real time detection of micro-organisms including resistant bacteria, the treatment and prevention of infections, as well as basic and behavioural research.

Antimicrobial resistance is a complex problem that requires co-ordinated action involving all stakeholders along the food supply chain. The One Health approach acknowledges the interconnections among the health of humans, animals and the environment, and the need for collaborative efforts across these sectors.

\section{Findings}

Over the last decade or so, Canada has made significant progress in building an evidence-based approach to assessing the antimicrobial resistance risk from antimicrobials licensed for use in animals. Some examples include: the categorizing of antimicrobials into four categories based on their importance in human medicine to help prioritize risk management options, and to support microbiological safety evaluation of veterinary antimicrobial drugs by using data from the Canadian Integrated Program for Antimicrobial Resistance Surveillance (CIPARS). In creating this list of medically important antimicrobials (MIAs), Health Canada considers the WHO's List of critically important antimicrobials within the Canadian context. More specifically, the use of MIAs are limited to the treatment and prevention of disease, and should not be used for growth promotion or for long, unspecified time periods in the treatment of animals.

Specific actions to increase oversight and promote the responsible use of antimicrobials in animals include the implementation of specific regulatory (2017) and policy changes (2018/2020). For example:

- Mandatory reporting of MIA sales information by manufacturers, importers, and compounders to support antimicrobial use and resistance surveillance in Canada.

- Ensured that all MIAs are only available under prescription status with veterinary oversight to ensure responsible use.

- Launched a risk-based post-market re-evaluation framework for MIAs to ensure the use of MIAs in animals do not pose an AMR risk for humans.

The following summary findings are key examples meant to highlight some of the work being undertaken under the four pillars; surveillance, stewardship, infection prevention and control, and research and innovation, of the Tackling Antimicrobial Resistance and Antimicrobial Use: A Pan-Canadian Framework for Action.

\section{Surveillance}

Under PHAC's leadership, CIPARS monitors AMU and resistance in enteric bacteria in humans, animals and food, with a focus on bacteria and antimicrobials of public health importance. The CIPARS program has been conducting surveillance since 2002. Each year an extract of CIPARS findings is included in the Canadian Antimicrobial Resistance Surveillance System (CARSS) report; this report combines data from all the Public Health Agencies of Canada AMR and information from the AMU surveillance programmes, including the use of antimicrobials in human medicine.

Resistance surveillance: For human health, Salmonella isolates/data provided to CIPARS are the product of an enhanced, passive surveillance collaboration with provincial public health laboratories. Human Campylobacter isolates are acquired through FoodNet Canada, a sentinel-site based food-borne disease surveillance system linked to CIPARS. For food and animals, CIPARS has active surveillance in which Salmonella, Campylobacter and generic Escherichia coli are recovered from grocery store meat, from healthy animals at slaughter and from healthy animals on sentinel farms. CIPARS has active sentinel surveillance for broiler chickens, turkeys, and grower-finisher pigs. Recently, new national farm surveillance pilot programmes have been launched for feedlot beef and dairy cattle.

Resistance findings: Overall, ceftriaxone resistance in Salmonella and E. coli isolates from chicken sources remained relatively stable or decreased after the 2014 poultry initiative to eliminate the use this antimicrobial class (i.e. drug is ceftiofur) for disease prevention purposes. Since 2015, there has been no reported ceftiofur 
use in sentinel broiler chicken flocks. In 2018, CIPARS observed small numbers of S. Enteritidis isolates resistant to nalidixic acid from chicken(s) (resistance not previously observed in poultry in Canada), and there were small numbers, but more isolates resistant to 6-7 classes of antimicrobials.

Antimicrobial use and antimicrobial sales surveillance: CIPARS farm surveillance involves administration of questionnaires to capture information on antimicrobial use, disease, and farm management practices (including biosecurity practices and vaccination). Information on quantities of antimicrobials distributed for use in animals were previously provided to CIPARS through a voluntary partnership with the animal health product trade association. Data on antimicrobials sold for use as pesticides on food crops are provided by Health Canada's Pest Management Regulatory Agency. Fisheries and Oceans Canada has data available on AMU for all aquaculture operations. Data on the quantities of antimicrobials dispensed at community pharmacies or purchased by hospitals (from IQVIA; accessed via the Canadian Antimicrobial Resistance Surveillance System) are also reported. As of the 2017 regulatory amendment, Health Canada's Veterinary Antimicrobial Sales Reporting (VASR) system collects volumes of antimicrobial drugs sold or compounded for veterinary use; this will replace the previous data provided to CIPARS by the animal health product trade association. The data on sales of antimicrobials should be interpreted carefully, as they may not reflect the actual patterns of animal use, and should be considered in conjunction with end-user information.

Antimicrobial use and antimicrobial sales findings: The overall quantity (kgs) of antimicrobials (excluding ionophores and coccidiostats) distributed for sale decreased by $12 \%$ between 2009 and 2018 . Over the same period, the quantity of fluoroquinolones increased by $79 \%$ (from $377 \mathrm{kgs}$ to $677 \mathrm{kgs}$ ). However, when the data are adjusted for the biomass of the animal production (PCU), the total quantity of antimicrobials distributed for production animals fell by $7 \%$ between 2009 and 2018. In 2015, the quantity of antimicrobial drugs distributed for use in animals peaked, but showed a marked decrease in 2016 and in 2017. Between 2017 and 2018, there was a 6\% increase in the amount of antimicrobials (taking into account the number of animals and their weights) distributed for use in animals, from information reported by the animal health product trade association. In contrast, between 2017 and 2018, the CIPARS sentinel farm data showed that there was an overall decrease in reported antimicrobial use on broiler chicken and grower-finisher pig farms, and an increase on turkey farms. In terms of the quantity of antimicrobials $(\mathrm{kg})$ intended for use in farming in 2018 , about $78 \%$ of antimicrobials were sold for use in production animals (food animals and horses), $21 \%$ for humans, about $1 \%$ for companion animals, and a negligible amount for use in crops (CAHI, IQVIA and Health Canada, Pest Management Regulatory Agency).

\section{Stewardship}

As of 1 December 2018, all MIAs now require a prescription with veterinarian oversight. In addition, MIAs can no longer be sold for growth promotion in food animals. The Canadian List of MIAs is broadly aligned with the WHO List. Moreover, livestock producers have adopted measures within their own sectors to improve antimicrobial stewardship. For example, the poultry industry has eliminated the use for preventative purposes of antimicrobials considered in Canada to be of very high, or high importance to human medicine, such as the third generation cephalosporins.

\section{Infection prevention and control}

Preventive approaches such as improved biosecurity are designed to reduce infection pressure and mitigate the transmission of pathogens, using a combination of procedural and physical barriers. Such activities, in combination with other preventive measures such as vaccination, and health monitoring and surveillance, may reduce the need for anti-infective medications, and foster their prudent, risk-based use. Since 2009, national biosecurity standards have been published for the major terrestrial animal agricultural commodities, in collaboration with industry associations and other stakeholders. They are tools to assist producers and industry and industry associations in developing and implementing farm level biosecurity plans, and governments in developing biosecurity programs to manage disease risks.

\section{Research and Innovation}

As of the 2017 Regulatory Amendment, Health Canada's Notification Program facilitates access to low risk veterinary health products (VHPs), such as vitamins and minerals to promote health and welfare of animals. 
The programme provides additional health management tools, and may also reduce the need to use antimicrobials.

In addition, through the Genomics Research and Development Initiative (GRDI), the Government of Canada is funding research to identify targets for action through mapping the development and transit of antimicrobial resistance through healthcare, animals, plants, and the environment to preserve the effectiveness of the antimicrobials that Canadians rely on every day. Genomics, approaches (metagenomics, whole-genome sequencing, genomic epidemiology) will be applied to the study of AMR dissemination through the One Health continuum to identify hot-spots of AMR transmission and to inform strategies for reducing the spread of AMR in Canada. For instance, as part of the GRDI AMR project, federal researchers are using Whole Genome Sequencing to assemble detailed genomic profiles of thousands of bacterial isolates collected from a variety of environments over a number of years. Based on analysis of the data, researchers are bringing a new clarity to our understanding of how AMR can be transmitted, in particular, the role played by plasmids.

\section{Summary}

Canada takes a co-ordinated One Health approach to AMR across the Federal/Provincial/Territorial governments including all stakeholders, such as animal producers, industry, veterinarians, and researchers. The Federal Government continues to focus efforts on four key areas; surveillance, stewardship, infection prevention and control, and research and innovation initiatives. Provincial and Territorial Governments continue to regulate the use of antimicrobials and introduce complementary actions to combat AMR. These initiatives represent a positive starting point in combatting AMR/AMU in the Canadian context, and will help improve AMR interventions across the agri-food sectors.

\section{Denmark}

Denmark is a large producer and exporter of livestock and livestock products, especially pigs and pig meat. Since the 1970s, pork production has increased sharply and this increase was initially accompanied by a rise in the use of antimicrobials in production. However, over the last 20 years, the use of antibiotics in pig production (which accounted for about $75 \%$ of antibiotic usage in 2018) has fallen steadily, while production has continued to grow. In 1998-2000, Denmark phased out the use of antibiotics for growth promotion (AGPs) in food producing animals. This change was accompanied by an increase in public and private investment in disease prevention measures such as improved hygiene, nutrition, breeding, and animal housing. These changes led to a significant reduction in the use of antibiotics in animal production. In 2017, approximately 94 tonnes of antibiotics were used by food producing animals (39mg/PCU). Denmark now ranks amongst the lowest of the 31 European countries reported in the ninth ESVAC Report (EMA 2019), and the OIE Annual Report (4th) on Antimicrobial Agents intended for Use in Animals.

Denmark has placed a high priority on tackling the rise in antimicrobial resistance and the use of antimicrobials in food producing animals. While the current level of antibiotic usage in livestock production is low by international standards, stricter targets were introduced to reinforce this downward trend. The use of antibiotic drugs in both humans and animals are by prescription only, and the national regulations on antibiotic usage are strictly enforced. The current Danish One Health Strategy against Antibiotic Resistance (2017) was developed based on the experience from earlier initiatives to combat the rise in resistance. The adoption of two regulations, the National Antibiotics and Resistance Action Plan (2010), and the European Commission's recommendations on AMR (2012) have contributed to reducing the need for antibiotics in animal production. Furthermore, the Nordic Declaration on Antimicrobial Resistance, adopted in 2015, has also had an important impact on combatting the emergence and spread of antibiotic resistance.

In 2010, Denmark's National Plan on antimicrobial resistance with a One Health focus was adopted. The implementation of this plan contributed to a $24 \%$ reduction in the use of antibiotics in animal production between 2009 and 2018. In 2013, Denmark introduced taxes on certain antibiotics at a rate of $11 \%$, primarily on fluoroquinolones and third and fourth generation cephalosporins. The revenue from this tax is used to fund various initiatives that are aimed at reducing the use of veterinary antimicrobials. In 2017, the Ministry of Environment and Food together with the Ministry of Health launched the second National Plan on the One Health Strategy to Combat Antimicrobial Resistance (DMH \& MEFD) with objectives similar to the Global Action Plan. 
The 2017 Strategy sets out a framework for improving the co-ordination of actions across human health, animal health and the environment with a view to reducing the incidence of AMR. Specific targets are set out for the use of antibiotics in animals and these are implemented as part of the strategy.

\section{One Health Strategy against antimicrobial Resistance 2017}

The current strategy on AMR focusses on five central objectives to combat the rise in antibiotic resistance and to reduce the use of antimicrobials in human health and animal health. The five broad goals are:

- A prudent use of antibiotics to reduce the incidence of resistance.

- Greater efforts to prevent infections and to facilitate antibiotic alternatives.

- Enhanced knowledge to improve targeted measures.

- Information and guidance on resistance and transmission.

- Closer international cooperation to minimise the development of antibiotic resistance.

In Denmark, the primary focus is to reduce the overall use of antibiotics in food animal production, especially the use of the highest priority critically important antibiotics, in order to achieve a low level of antibiotic resistance. There are strong restrictions on the use of antibiotics in human medicine, and approximately $90 \%$ of the over-all use of antibiotics in human medicine are prescribed in primary healthcare services. The overall use of antibiotics in livestock production is low following the implementation of tighter regulations and specific measurable targets on the use in animal production. Moreover, a multi-annual monitoring programme has contributed to this decline in the use of antibiotics for food producing animals.

Another feature of the AMR strategy focuses on preventing the transmission of infections between different animal species, and between animals and humans. More specifically, there has been a strong emphasis on improving livestock management, hygiene, disease diagnosis and the use of vaccines to prevent disease and AMR infections in food producing animals. For example, in the diary sector vaccines are used to prevent mastitis in dairy cows. A strong focus on hygiene along the food chain and specifically at slaughterhouses contributes to a reduction of AMR in food. This is achieved through action plans for zoonosis reduction in the production of broiler chicken, beef and pork. The current strategy emphasizes efforts to acquire robust scientific knowledge on the use of antibiotics and the development of resistance through sound and systematic surveillance systems. Since 1995, the reporting of data on antibiotic use and resistance in animals and humans has been under the responsibility of the Danish Integrated Antimicrobial Resistance Monitoring and Research Programme (DANMAP).

The National Antibiotic Council was established in 2010 to oversee all activities on antimicrobial use and resistance in human and animal health. In Denmark, antibiotics can only be obtained by prescription for the treatment of human or animal infections. Furthermore, a high priority is given to Danish participation in international forums on finding alternative solutions to antibiotics in the prevention and treatment of disease. The Danish strategy places emphasis on communications and training and livestock producers are encouraged to adopt preventative measures, in particular, good on-farm and off-farm biosecurity measures, and management practices. A combination of regulations and open communications among Government, veterinarians and livestock producers have been an important feature to ensure compliance with the targets set out in the national strategy.

The primary objective of the Danish Veterinary and Food Administration (DVFA) is to promote food safety and quality along the food chain from the producer to the consumer. In addition, the DVFA is also responsible for ensuring regulatory compliance on veterinary medicines through the supervision, guidance and control of the supply of animal medicines to veterinarians and farmers. All veterinary antimicrobials are under prescription only, and administered under veterinary supervision. The prophylactic use of antimicrobials is no longer permitted in food animals under Danish Law and antimicrobials can only be used for therapeutic and metaphylactic purposes. The herd level data collection system of AMU (VetStat) is seen as a useful model for many European countries (see www.aacting.org). This system allows for the measurement of AMU at herd level and for each herd to be compared to the average (benchmarking). In this way, every farmer and veterinarian is responsible for optimizing the use of antibiotics. Moreover, this system allows for the identification of herds that are high users of antibiotics, and identifies the actions that are needed in these 
cases. In Denmark, a transparent system is critical in order to optimize the use of antibiotics on livestock farms.

\section{Findings}

Since the introduction of regulations on antibiotic use in 2002, and with the additional changes in 2016, the use of fluoroquinolones has fallen sharply in Danish agriculture (DTU Food 2018). In 2010, the DAFC requested veterinarians to stop the prescription of third and fourth generation cephalosporins and, consequently, their current use is now negligible. Also in 2010, the Yellow Card Initiative (YCI) on the use of antibiotics in the pig sector was introduced, and this initiative has led to decreased antimicrobial use in pig production. In essence, the $\mathrm{YCl}$ sets a threshold for the consumption of antibiotics in a pig herd. If the threshold is exceeded, the farmer receives a warning. The use of colistin was heavily restricted due to the implementation of the $\mathrm{YCl}$, as well as the fluoroquinolones and the third and fourth generation cephalosporins. However, with the sharp decline in antibiotic usage in pig production, the sales of vaccines rose sharply from 28 million doses in 2009 to 60 million in 2018 (DVFA).

The strong emphasis on the prevention of disease at farm level has been a central plank of the strategy on AMR. In particular, there has been a special focus on three aspects; farm biosecurity measures, targeted use of vaccines and improved diagnostic tests adapted to the specific herd. In 2010, the Danish National Antimicrobial Council was established to promote and implement the One Health plan to tackling antimicrobial resistance. A further objective of the Council is to identify alternatives to antibiotics in treating and preventing infections, and to provide guidance to national institutions on antimicrobial use and resistance.

Denmark has extensive experience in implementing a One Health approach to tackling antimicrobial resistance. Prescription guidelines on antibiotics have been developed and are strictly implemented to achieve the optimum clinical effects of treatment in conjunction with improving on-farm animal husbandry practices. In an effort to enhance evidence based advice for the government on antimicrobial use and resistance, an Advisory Committee on Veterinary Medicines(ACVM) was established (2018). One of the first tasks for the Committee was to give advice on a measurable target for antimicrobial use in pig production. Since 2010, the initiative has been successful in reducing the use of antibiotics in the pig sector, and has shown no adverse impacts on the welfare of the animals, or on the profitability of the farms (Aarestrup et al., 2010).

\section{Summary}

Denmark was one of the first countries to adopt a holistic or One-Health approach to tackle antimicrobial resistance. Danish policies on AMR have continued to evolve in response to the availability of better scientific evidence on resistance and better data on the use of antibiotics in food producing animals. A number of innovative policies have been adopted and have proven successful in reducing the use of antibiotics in pig production, including; the setting of specific targets for antibiotic use in food producing animals, the development and implementation of the $\mathrm{YCl}$ (which targets pig farms with the highest levels of antibiotic use). The Advisory Committee on Veterinary Medicines plays an important role in providing guidance on antimicrobial use in livestock and gives advice on new national measurable targets for a reduction in antimicrobial use in the pig sector 2019-2022. Overall, the use of antimicrobials in livestock production has fallen by $24 \%$ between 2009 and 2018. While good progress have been made, a new national target has been set to reduce antimicrobial use in the pig sector by a further $8 \%$ by 2022 (compared to 2018).

The Danish approach to tackling AMR includes greater emphasis on the prevention of animal diseases, herd level data collection and benchmarking, as well as open and extensive consultations with all stakeholders along the food chain. In addition, establishing reliable institutions for the surveillance and data collection on antimicrobial use and resistance, and the enforcement of the national regulations on antimicrobial usage in livestock agriculture. Policies to improve animal health and welfare have tended to concentrate on improving internal and external farm biosecurity measures and the selective use of vaccines. This systematic approach to tackling AMR at farm level has facilitated a relatively smooth adjustment in production and has contributed to maintaining animal productivity and farm incomes, while keeping the adjustment costs modest at farm level. 


\section{Ireland}

The use of antimicrobials in food producing animals in Ireland is low by international standards due to the type of animal production system and the large proportion of ruminants in the animal population. The Health Products Regulatory Authority (HPRA) collects data on the use of veterinary antibiotics in Ireland, based on sales data. Overall, approximately 100 tonnes of veterinary antibiotics are sold annually for food animals, but this figure has trended downwards in recent years. While these aggregate figures are useful for broad international comparisons, the detailed breakdown of antibiotics use by species and farming system are not yet available. As part of the National Action Plan on AMR, a new consumption database is ongoing, and this database will provide granular information on the individual animal sector consumption of antibiotics from 2022.

\section{National Action Plan on AMR (iNAP)}

Ireland's National Action Plan on Antimicrobial Resistance 2017-2020 (iNAP) was implemented in 2017 to tackle the increasing threat posed by antimicrobial resistance. Like many countries, the iNAP takes a multisectoral One Health approach to tackling the causes of AMR working with stakeholders across the different sectors. The National Interdepartmental AMR Consultative Committee was established to co-ordinate this work and to encourage engagement of all stakeholders across the different sectors. The Chief Medical Officer and the Chief Veterinary Officer jointly lead this Committee. The iNAP was developed by the Department of Health and the Department of Agriculture with oversight from the Consultative Committee. This plan has built on the existing measures in each sector to combat AMR, and brought these together under the One Health framework. The overall goal of iNAP is to reduce the quantity of antibiotics consumed in human medicine and in the animal sector in Ireland.

The iNAP sets out a range of policies to prevent, monitor and combat AMR across the health, agriculture and environmental sectors. The key strategic interventions for tackling AMR are in line with the GAP on AMR across the three sectors. In Ireland, antimicrobial resistance has been classified as a national risk and has been included in the National Risk Assessment (NRA) framework since 2014. This framework acknowledges the threat due to the emergence and spread of resistant bacteria and the increasing ineffectiveness of antibiotics in treating human and animal diseases.

Since 2000, significant changes in the legislation have been introduced on the use of antimicrobials in animals. These measures were further strengthened in 2014 when the Department of Agriculture, Food and Marine (DAFM) introduced a national surveillance programme for AMR. Since 2007, Ireland, like other European Union States, has taken a harmonised approach to monitoring antimicrobial resistance in poultry and pigs since 2007 based on Directive 2007/407/EC. Prior to this, Directive 2003/99/EC was the legal basis for the monitoring of antimicrobial resistance in animals and indicator organisms isolated from animals and food products.

The DAFM co-ordinates the AMR surveillance system in line with EU legislative requirements. The Central Veterinary Research Laboratory, as the National Reference Laboratory carries out the analyses of samples submitted for isolate testing. The results of the isolate testing are sent to the Food Safety Authority of Ireland, who collates the data and then sends it to the European Food Safety Authority (EFSA).

Moreover, the DAFM is also responsible for the maintenance of the legislation in all areas relating to the manufacture, supply and use of veterinary medicines including antibiotics in Ireland. Specific controls on the manufacture, supply and use of all veterinary medicines are under EU legislation dating back to the 1990s. In Ireland, all veterinary medicines must be authorised by either the Health Products Regulatory Authority (HPRA), or the European Medicines Agency (EMA).

All veterinary antibiotics are classified as prescription only medicines, while the use of antibiotics for growth promotion in animals was banned in 2006. The national legislation for the control of veterinary medicines and the production of medicated feeds is based on two EU directives (Council Directive 2001/82/EC and Council Directive 90/167/EEC). In addition to monitoring compliance with the legislation and implementing of AMR surveillance, DAFM is also responsible for raising awareness of AMR and makes recommendations on the actions to be taken by the different stakeholders.

The goal of iNAP is to ensure the availability of effective antibiotic treatment options for both humans and animals. More specifically, the iNAP aims to: 
- Improve awareness and knowledge of AMR. This is to be achieved through education and information campaigns, intelligence and data collection.

- Enhance surveillance of antibiotic resistance and antibiotic use. To improve surveillance systems that facilitate greater standardisation of data collection, data linkages and sharing of real time information.

- Reduce infections and the spread of disease. Efforts to improve the prevention and control of disease, include national guidelines and standards in relation to hygiene and biosecurity practices.

- Optimize the use of antibiotics in human and animal health. Through the development and implementation of antimicrobial stewardship programmes, the promotion of prudent prescription of antimicrobials, and access to rapid diagnostics.

- Promote research and sustainable investment in new medicines, diagnostic tools, vaccines and other interventions. Through measuring and evaluating costs of AMR, identifying research opportunities and working with key stakeholders to develop alternatives to treating animal diseases.

For each of the five objectives outlined above, the iNAP sets out a detailed roadmap for the implementation, including the strategic interventions, activities, responsibility, as well as the timeline for each component of the plan. In addition, the plan identifies the interventions and activities that are cross sectoral, and those that are sector specific over the lifetime of the plan. The National Interdepartmental AMR Consultative Committee has the overall responsibility for monitoring the implementation of iNAP. The implementation of the animal health component of the plan was assessed bi-annually over the period 2017-2020. The Committee monitored the progress with the implementation of specific actions in all three sectors; health, animal and the environment, and provided advice and guidance in relation to concerns that have emerged.

A range of measures have been taken by animal producers to mitigate the emergence and spread of AMR, including the implementation of better disease control programmes and bio-security measures which are aimed at reducing the need for antibiotics in animal production. However, in some sectors the volatility and downward pressure on livestock prices has reduced incentives for producers to invest in measures to lower the risk of AMR infections. In terms of overall expenditure on veterinary medicines, the Irish Farmers Association estimate that livestock farmers spent about EUR 176 million annually on veterinary medicines; of which, an estimated EUR 57 million are for antimicrobials.

The overall amount of antibiotics sold in Ireland is estimated at $48 \mathrm{mg} / \mathrm{PCU}$. This places Ireland well below the average use in EU Member States. According to the HPRA, more than $70 \%$ of antibiotics administered to food animals are in the feed or in the drinking water. However, a more systematic collection of farm antimicrobial use data would help to better understand the trends in the different food animal sectors. Livestock producers have been encouraged through public awareness and training campaigns to take a holistic approach to biosecurity and animal husbandry, involving an integrated approach to disease prevention and control in order to combat AMR.

One of the main drivers of AMR is the overuse of antibiotics in animal production. To address this issue Ireland has focused on optimising the use of existing antibiotics. An important objective of iNAP is the development of an integrated national surveillance system to provide consistent coverage of antibiotic use in all animal sectors. In November 2019, the DAFM introduced an antimicrobial consumption database for pigs, which will collect farm level quarterly data on antimicrobials use.

\section{Findings}

Over 500 veterinary antibiotic medicines are currently authorised for use in Ireland, including medicines authorised by the HPRA, as well as those authorised by the EU Commission. This includes 50 individual antibiotic substances. These data are based on self-declarations by applicant companies (MAH), and do not include other veterinary antibiotics that are authorised under special licence, and human antibiotics, that may be used in the treatment of specific animal diseases. The total volume of veterinary antibiotics sold in Ireland in 2017 was 99.7 tonnes. A small number of antibiotic classes, namely tetracyclines (41.6\%), penicillins $(20.9 \%)$, sulphas and trimethoprim (18.1\%), and macrolides and lincosamides $(7.5 \%)$, account for almost $90 \%$ of total sales. 
In Ireland, sales of the different classes of antibiotics have remained relatively stable over the period 20132017. However, there is some concern regarding the increasing trend in the sales of $3^{\text {rd }}$ and $4^{\text {th }}$ generation cephalosporins and macrolides. In general, smaller quantities of these antibiotics are required to treat diseased animals, and thus they represent a relatively small percentage of the overall sales. However, this trend towards the use of more potent classes of antibiotics can be hidden within the overall figures.

Fluctuations in the year-to-year sales data of antibiotics can be due to several factors including, the seasonal prevalence of disease, the precise timing of the end-of-year transactions, the quantity of antibiotics held in storage, changes in the size of the national herd, exports of veterinary medicines and changes in the class of antibiotics being prescribed. ${ }^{4}$ In November 2018, DAFM set out specific conditions under which the High Priority--- Critically Important Antibiotics (HP--ClA) may be used in veterinary medicine. In essence, the HPCIAs should not be used for disease prevention, but can be used under certain conditions where there are no alternatives available to treating the infection.

Regarding farm level data on antibiotic use, a recent study by Earley et al. (2019) on the use of antimicrobials in calves reared on dairy and beef farms attempted to quantify the use of antimicrobials, as well as the classes of antimicrobials used. This study provided detailed information on farm usage of antimicrobials in suckler beef and dairy calves from birth to six months of age. A total of 123 farms (79 beef and 44 dairy) representing 3204 suckler beef calves and 5358 dairy calves were included in the study. The study suggested actions that farmers can do to optimize the use of antimicrobials in animal production, For example, the study advocated using antimicrobials only under veterinary supervision, using the correct dosage, avoiding more potent antimicrobials, using vaccines, improving biosecurity and avoiding the use of antimicrobials to treat viral diseases.

\section{Summary}

In summary, while it is too early to draw definite conclusions on the impact of the iNAP, some preliminary findings and insights can be drawn from this work. These findings show that the implementation of iNAP has raised public awareness of the direct and indirect impacts of AMR on animal, human, and environmental health. In addition, it has also helped to focus efforts on improving the integrated surveillance system on antibiotic use in animals and, in particular, the need for greater efforts to collect farm level data on the different food animal species. Finally, the findings suggest that greater efforts are needed to improve animal health and welfare by enhancing farm biosecurity measures, and by adopting good animal husbandry practices.

\section{Japan}

In Japan, the Ministry of Agriculture, Forestry and Fisheries (MAFF) is responsible for animal health and livestock animal welfare, but not for hygiene along the food chain at, and after, the abattoir stage. Much of the legislation in Japan on antimicrobial use is based on risk analyses and this work is carried out by the regulatory authorities. In practice, each risk management option is assessed in relation to human health and animal health and welfare. The Food Safety Commission (FSC) is responsible for assessing the risks to human health associated with the transmission of antimicrobial resistance from animal products through the food chain. MAFF has developed guidelines to determine feasible risk-management options based on the assessment of risks arising from the use of veterinary antimicrobials and antimicrobial feed additives in food producing animals in 2012 and 2013, respectively. The latter guidelines were updated in 2017.

The Japanese Veterinary Antimicrobial Resistance Monitoring System (JVARM) was established in 1999, and its main goal has been to monitor AMR in livestock production. The results of this monitoring process enables an assessment of the trend in the emergence of antimicrobial resistant bacteria and the appropriate risk management measures. The agricultural monitoring system collaborates with the human healthcare sector and exchanges useful data for establishing a comprehensive antimicrobial resistance monitoring system across human health and animal health.

Various measures have been taken to ensure that antibiotics are used appropriately in animal production including, the setting of sector specific norms and standards in line with the national legislation. Furthermore,

\footnotetext{
${ }^{4}$ The impact of Brexit is uncertain as the negotiations between the European Union and the United Kingdom are ongoing.
} 
the government has also developed guidelines on the prudent use of veterinary antibiotics in order to ensure their optimum use for therapeutic purposes and minimises the emergence of antimicrobial resistant bacteria.

\section{National Action Plan on Antimicrobial Resistance 2016-2020}

Japan's National Action Plan on Antimicrobial Resistance (NAP) was published in 2016 and covers the period 2016 to 2020. The NAP is largely based on the strategic objectives as set out in the Global Action Plan on AMR. Prior to the implementation of the NAP, which adopts a One Health approach to tackling antimicrobial resistance; extensive regulatory measures have been in place for antibiotic use in human medicine and in veterinary medicine. In addition, the NAP includes an objective on international co-operation in tackling AMR. An important feature of the NAP is that specific numerical targets are set for certain resistance rates by 2020 .

The NAP outlines a range of measures to minimise the emergence of AMR and prevent the spread of infectious diseases caused by antimicrobial resistant organisms. For many years, the different Ministries and Agencies have implemented measures against AMR in their respective areas. However, since 2016 the relevant Ministries have been working together in a more integrated manner under the NAP. The overall coordination is under the responsibility of the Cabinet Secretariat (CAS). Since the 1990s, Japan has implemented various measures to combat the rise in AMR, and this experience has been used to prioritize the measures set out in the five-year plan 2016-2020.

In Japan, the control of AMR in livestock production and aquaculture is implemented based on the risk analysis principles as defined by the OIE and the Codex Alimentarius Commission. Based on the outcome of the scientific risk assessment and the on-farm situation, risk management measures are implemented in line with the estimated risk level. The NAP is structured around the following goals to:

- Improve public awareness and understanding of AMR, and promote education and training of professionals.

- Monitor antimicrobial resistance and use of antimicrobials as well as the trends and spread of AMR.

- Implement appropriate infection prevention and control measures to prevent the spread of antimicrobial resistant bacteria.

- Promote the appropriate use of antimicrobials in healthcare, livestock production and aquaculture.

- Promote research and development on antimicrobial resistance and identify better measures to prevent, diagnose and treat antimicrobial resistant infections.

- Support international cooperation and enhance global multidisciplinary measures to contain antimicrobial resistance.

The activities and strategies to combat AMR are set out in detail in the NAP, as well as the numerical targets to be reached by 2020. Substantial efforts have been made to reduce the use of broad-spectrum antibiotics, with improved diagnostic tests, targeting and the prudent use of appropriate antibiotic classes to deal with infections in humans. The NAP does not set specific targets for reduction of antimicrobial use in foodproducing animals. Instead, it sets targets for the reduction or maintenance at a low level, of the resistance rates in food-producing animals. The real goal is to reduce the negative effect on human health of resistant bacteria, while ensuring that antimicrobials remain effective in preserving human and animal health. Accordingly, it is appropriate to use indicators that are much more closely tied to that goal, namely, figures showing the distribution and levels of antimicrobial resistance in certain particular bacteria.

The NAP targets for food-producing animals have been set for tetracyclines, third-generation cephalosporins and fluoroquinolones resistance of Escherichia coli. As in many countries, tetracyclines are the most commonly used antimicrobial in food producing animals in Japan. Therefore, resistance to tetracycline is one of the most appropriate indicators to monitor as to whether risk-management measures are effective. Third-generation cephalosporins and fluoroquinolone are critically important for both human and animal health. Therefore, stricter risk-management measures are taken for these antimicrobials, such as their prohibition in cases where other veterinary antibiotics are effective, and mandatory periodic post-marketing reporting concerning the emergence of resistant bacteria. Thus, the rates of resistance to third-generation cephalosporins and fluoroquinolones among Escherichia coli are also chosen as numerical indicators. The figures for these indicators in Japan have been at around the same low levels as in other G7 countries at 
the time of establishing the NAP. Essentially, the targets are to maintain them at the same low level as in other $\mathrm{G} 7$ countries until 2020, assuming that other $\mathrm{G} 7$ countries maintain them at low levels by implementing their own action plans.

\section{Findings}

In 1999, the Government of Japan established JVARM - a system to monitor nationwide trends of antimicrobials resistance in livestock. According to JVARM results, the rate of tetracycline resistance of Escherichia coli in livestock fell from $59 \%$ in 2001 to $40.8 \%$ in 2017 . This was partly achieved through measures for ensuring appropriate antimicrobial use in animal production. The rates of resistance to thirdgeneration cephalosporins (in the range of $0.5 \%-6.8 \%$ ) and fluoroquinolones (in the range of $0.9 \%-5.2 \%$ ) among Escherichia Coli have been kept at low levels from 2001 to 2017.

The manufacture and importation of veterinary antimicrobials for therapeutic use are covered under the 1961 Regulations for the Control of Veterinary Pharmaceutical Products and annual reports are sent to MAFF. Overall, there are 13 classes of veterinary antibiotics used in food animal production. Data on the sales of veterinary antimicrobial agents and the target species are published by MAFF. Over the period 2009 to 2018, the quantity of veterinary antimicrobials sold for therapeutic purposes in animals fell from 854.5 tonnes (active substance) to 824.6 tonnes, or by about $4 \%$. The most well used classes of antimicrobials are tetracyclines at $44 \%-38 \%$, sulphonamides at $17 \%-11 \%$, penicillins at $11 \%$ to $13 \%$, macrolides at $9-19 \%$, and aminoglycosides at $6 \%$ to $4 \%$. The high usage of tetracyclines is attributed to their widespread use for respiratory diseases and relatively low cost.

The regulations on antimicrobial use are closely linked to the risk assessment of the technical, economic, environment and social risks associated with the different classes of antibiotics in the different sectors. More specifically, for food producing animals (pigs, chickens, cattle, others), the estimated quantity of veterinary antimicrobial sales fell by about $11 \%$, between 2009 and 2018, and ranged from 721 tonnes in 2009, to 646 tonnes in 2018. Regarding the sales of the different classes of antibiotics, the sales of tetracyclines accounted for the largest share, at $40 \%$ of the total, while third generation cephalosporins and fluoroquinolones accounted for less than $1 \%$ of total sales.

The fall in antimicrobial sales to animal producers for inclusion in feed is modest. More specifically, the volume of antimicrobial feed additives fell from 233 tonnes to 221 tonnes over the period 2009 to 2018 . As regards the antimicrobial classes, polyether has showed an increase of $54 \%$, while most of the other antimicrobials fell, including tetracyclines, which fell by $100 \%$. This trend is unlikely to be reversed because the designations of antimicrobials as feed additives, such as Virginiamycin (2018), Colistin Sulfate (2018), Tylosin Phosphate (2019), tetracyclines (2019), were successively revoked based on the results of risk assessment conducted by the Food Safety Commission, and is in line with the objectives of the NAP.

In terms of species, the largest amount of antibiotics was sold to the pig sector (541 tonnes in $2009-486$ tonnes in 2018), followed by broiler chickens (66-65 tonnes), dairy cattle (39-41 tonnes) and beef cattle (3133 tonnes) from 2009 to 2018. Over this period, antibiotic usage for pigs was about ten times higher than for other food-producing animals. Concerning the highest priority critically important antimicrobials, the percentage of cephalosporins (0.06- 0.12 ) and fluoroquinolones $(0.31-0.39)$ in total sales intended for use in pig production were low, while that of macrolides $(4.1-11.88)$ increased over the same period.

\section{Summary}

The National Action Plan on Antimicrobial Resistance 2016 -2020, brings together under a single framework the measures to tackle AMR in the different animal sectors. The national policies and regulations on AMR are underpinned by an extensive system of risk analyses and risk assessment including technical, economic, environmental and social risks. This approach has enabled Japan to implement strict regulatory measures on the use of antibiotics supported by a robust and integrated surveillance system. This rigorous approach has enabled the collection of extensive and reliable data on antimicrobial resistance and antimicrobial use in food producing animals.

Japan is unique in setting antimicrobial resistance rates, and not on the quantity of antimicrobials used, as indicators of the success of the NAP. In terms of antimicrobial resistance rates among Escherichia coli, tetracycline resistance in Escherichia coli is showing a decreasing trend. This was achieved through measures that ensured the appropriate level of use of antibiotics in farm animals. At the same time, the rates 
of resistance to third-generation cephalosporins and fluoroquinolones among Escherichia coli have been kept at a low level. The specific risk management measures taken regarding food-producing animals for these critically important antimicrobials both for human and animal health are also showing favourable results.

While the reduction of antimicrobial use in food producing animals is not an indicator specified in the NAP, a modest overall fall in the total quantity of antimicrobial use has been observed, with small fluctuations in both directions. This is thought to be partly due to animal producers adopting alternative practices to antimicrobials in production.

\section{United States}

The US National Action Plan for Combating Antibiotic-Resistant Bacteria (2015-2020), focuses on several actions to reduce AMR including, improving the stewardship of antibiotics, increasing surveillance and monitoring, and encouraging investment in research and innovation of new disease preventative measures. The Plan was developed by the Interagency Task Force for Combatting Antibiotic Resistant Bacteria in response to Executive Order 13676 issues by President Obama in 2014. Essentially, the Plan is designed to guide actions by public health, healthcare and veterinarians in an effort to address the risks posed by AMR. More specifically, the Plan supports the five main goals identified in the US National Strategy for Combating Antibiotic-Resistant Bacteria emphasises the need to enhance collaboration amongst stakeholders across all sectors. In addition, the NAP outlines a series of steps to implement the Strategy while also addressing the policy recommendations from the Presidents' Council of Advisors on Science and Technology.

\section{National Action Plan for Combating Antibiotic Resistant Bacteria 2015}

The NAP sets out specific milestones for actions over the five-year period 2015 to 2020 , in order to optimize the use of antibiotics, reduce the incidence of antimicrobial resistance in human and animal health, to reduce inappropriate use, and to foster and support antibiotic stewardship.

Broadly, the NAP follows the framework outlined in the GAP and focuses on the five central goals to address the risks from antimicrobial resistance. More specifically, these goals are to:

- Slow the emergence of resistant bacteria and prevent the spread of antimicrobial resistant infections.

- Strengthen national One Health surveillance efforts to combat antimicrobial resistance.

- Advance development and use of rapid and innovative diagnostic tests for identification and characterization of resistant bacteria.

- Accelerate basic and applied research and development for new antibiotics, other therapeutics and vaccines.

- Improve international collaboration and capacities for antibiotic-resistant prevention, surveillance, control and antibiotic research and development.

The first objective focuses on the judicious use of antibiotics in healthcare and in animal production in order to slow the emergence of antimicrobial resistance, and to extend the lifetime of effective antibiotics. This goal also includes the optimal use of vaccines and other interventions to prevent infections and reduce the need for antibiotics, as well as more rapid diagnosis and control of disease outbreaks. A further objective emphasises the need to improve the integration of data from human and animal surveillance systems that monitor human and animal pathogens. This goal also involves enhancing the monitoring of antibiotic sales, usage, and resistance and management practices at multiple points along the food chain from farm to final consumer.

Another key objective focuses on the development of improved diagnostic tools for detection of resistant bacteria, while at the same time reducing the inappropriate use of antibiotics. There is strong emphasis on improving the accuracy of disease detection, as well as the speed of diagnosis in order to effectively manage any outbreaks of AMR infections. In addition, investing in research to develop new antibiotics and alternative treatments such as vaccines that can reduce the need for antibiotics is seen as an important longer-term 
part of the National Action Plan. Moreover, greater co-operation with other countries and international organisations is seen as a crucial element in the NAP to facilitate the sharing of experiences and best practices to prevent and control the emergence and spread of antibiotic resistance.

The National Action Plan however, does not set specific targets for antibiotic use in food producing animals, but sets targets for reducing antibiotic resistant bacteria in humans. For example, targets are set to reduce by $50 \%$ the incidence of overall Clostridium difficile infections by 2020 . The Task Force responsible for implementing the Strategies on AMR provides annual updates on the progress in implementing the NAP and the challenges that may arise during the implementation of the Plan.

\section{Findings}

The United States Food and Drug Administration (FDA) is responsible for collecting data on the annual sales of antibiotics, including sales for food producing animals. In the US, the sales of antibiotics for food animals reached a peak in 2015, but have fallen sharply in recent years. More specifically, over the period 2009 to 2015 , the total sales of the highest priority critically important antibiotics increased by $26 \%$ to reach almost 10000 tonnes. Moreover, the sales of cephalosporins increased by $61 \%$ over the period $2009-2015$, but sales data for the highest critically important third and fourth generation cephalosporins are not available for this period. The FDA withdrew one fluoroquinolone approval in 2001 and the other in 2005 because of the evidence that the use of fluoroquinolones in poultry led to an increase in fluoroquinolone-resistant Campylobacter infections in people. The sales of critically important fluoroquinolones antibiotics reached 20 tonnes in 2015; a one-third increase since 2013. According to the FDA, approximately $95 \%$ of farm antibiotics are administered (mass medication) in the feed or in the drinking water. As regard the nonmedically important antimicrobials such as ionophores, almost 5000 tonnes have been sold annually for use in animal production.

The 2018 FDA Report shows that the domestic sales and distribution of the highest priority critically important antibiotics for use in food producing animals fell by 33\% between 2016 and 2017, and by $43 \%$ since 2015 . On 1 January 2017, the FDA's new regulation on the use of medically important antibiotics in food producing animals came into effect and this may have contributed to this reduction. This regulation prohibits the use of antibiotics that are medically important for growth promotion or for improving feed efficiency in food animals. More specifically, the sales of medically important antibiotics used for growth promotion fell to negligible amounts in 2017.

Since 2015, the sales of the antibiotic classes most commonly used in food animal production in the United States have declined dramatically. More specifically, the sales of tetracyclines (which account for about twothirds of all antibiotic sales) fell by $40 \%$ between 2016 and 2017, while the sales of aminoglycosides, penicillins and macrolides fell by $19 \%, 18 \%$ and $15 \%$, respectively. However, the sales of fluoroquinolone antibiotics increased by $42 \%$. A report by Singer (2019), estimated the on-farm antimicrobial usage in broiler chickens and turkeys between 2013 and 2017. The report concluded that medically important in-feed antimicrobial drugs such as virginiamycin and tetracycline decreased by $60 \%$ and $95 \%$, respectively over the period, while the use of in-feed tylosin fell to zero. Moreover, the report also noted that there was a shift to non-medically important antimicrobials such as bacitracin and avilamycin in 2017 compared to 2013. Also, the medically important water soluble antimicrobials fell sharply, for example, water soluble penicillin, tetracycline and sulphonamides use fell by $21 \%, 47 \%$ and $72 \%$, respectively, over the period 2013 to 2017.

Regarding the different food animal sectors, the largest decline in antibiotic sales was recorded for broiler chickens, a 47\% fall between 2016 and 2017. This sharp decline can be attributed to several factors, including the increase in consumer demand for poultry meat that is produced with low antibiotics. As regards the other species, the sales of medically important antibiotics decreased by $35 \%$ for dairy cows and pigs. ${ }^{5}$ Overall, medically important antibiotics account for about $51 \%$ of antibiotics sold for use in food animals. According to the Natural Resources Defence Council (NRDC), the beef and pork industries remain high users of medically important antibiotics.

\footnotetext{
5 The sales report does not differentiate between types of cattle.
} 


\section{Summary}

The NAP covers the five year period 2015-2020, and focuses on the following areas for specific actions; the stewardship of existing antibiotics, improving surveillance and monitoring of antibiotic use and resistance, and investment in research on new antibiotics and alternatives to antibiotics in human and animal health. However, unlike some other countries, the NAP does not set specific targets on the use of antibiotics in food animal production. However, the NAP includes one target for the elimination of medically important antimicrobial drugs for growth promotion in animals, which fell to zero in 2017. The changes in the regulations on antibiotic use in animal production that have taken place in recent years have resulted in a steady fall in the sales of antibiotics in animal production.

Sales of antimicrobials for use in animal production reached a peak in 2015, but has fallen over the last five years. Moreover, the sales of the major antibiotics classes used in animal production have also fallen by $15 \%$ to $40 \%$ between 2016 and 2017 . The use of highest priority critically important antibiotics and medically important antibiotics have also declined and they are no longer permitted for enhancing animal growth. The most noticeable drop in antibiotic sales has been in broiler chicken production, as with the shorter life span (approximately six weeks); it is technically easier for less antibiotics in the production system.

\section{Brazil}

Brazil is a major producer and exporter of animal products, especially chicken, beef and pig meat. The poultry sector is large scale, intensive, and highly integrated, with about one-third of production destined for the export market. Beef cattle production, on the other hand, is largely based on extensive grazing systems. Antimicrobials are mainly used in the poultry and pig sectors. Brazil has a dual structure of production in the pig and poultry sectors, with the bulk of production destined for the domestic market and the remainder exported. For some food export markets, the use of antibiotics for growth promotion in animal production are prohibited. Brazil has an integrated animal production chain with close co-ordination between internal and external biosecurity measures. However, with the increasing pressure on animal health due to greater production intensity, interest in developing and implementing robust monitoring systems on antibiotic use in animal production has been growing. In recent years, changes to the regulations on antimicrobials in livestock production have resulted in the withdrawal of several antibiotics from use in animal production. Moreover, consumer concerns on the use of antibiotic growth promoters are increasing and this is resulting in growing demand for antimicrobial "free" meat.

In Brazil, antibiotics are used for therapeutic and prophylactic purposes, as well as for stimulating growth in animal production. While the amount of antibiotics used are not published officially, experts generally perceived that their use is declining due to changes in the production system and changes in consumer preferences. The intensive pig production systems use antibiotics in production, with some estimates indicating an average consumption level at $358 \mathrm{mg} / \mathrm{kg}$ of pigmeat produced (Dutra 2017). While antibiotics are still widely used as an additive in animal feed for managing animal health and growth promotion, there is growing research into alternatives such as probiotics and prebiotics. Livestock producers are particularly concerned over the potential increase in production costs if antimicrobials were unavailable for use in production.

The Health Surveillance Agency (ANVISA) is responsible for tracking the incidences of AMR in humans and food products and regularly publishes these results. The first regulations on the use of antimicrobials in livestock production were introduced in the 1960s and established the mandatory inspection over the use of veterinary medicines by the Ministry of Agriculture, Livestock and Food Supply (MAPA). All veterinary medicines produced in Brazil, or imported, must be licensed and registered with the Ministry of Agriculture, Livestock and Food Supply (MAPA). The Normative Instruction 65(MAPA 2006) regulates the use of veterinary drugs intended for use in animal feed. These regulations were modified in 2016 (Normative Instrument 14, MAPA 2016), and all antimicrobials administered via the feed with prophylactic or therapeutic purposes require a veterinary prescription. Some parts related to this Regulation will come into force in July 2021. Antimicrobials for growth promotion, however, are not yet included in this Regulation.

ANVISA, through Ordinance no 854/2016, created the Sanitary Surveillance Commission on Microbial Resistance (CVSRM). The purpose of CVSRN is to advise the Collegiate Board (DICOL) on health surveillance, standards and actions related to the monitoring, control and prevention of microbial resistance. CVSRM is a collegiate advisory body, technically linked to the Co-ordination of Strategic Programmes of 
SUS, with the objective of preparing and monitoring the implementation of the National Action Plan on Microbial Resistance. The update of RDC Resolution ANVISA No. 53/2012, in foods of animal origin, was included in the National Plan of Action on Resistance. Resolution RDC ANVISA no. 328/2019 was published after extensive discussions and contributions received through Public Consultation to update Resolution RDC Anvisa No. 53/2012, which established limits for only 24 active pharmaceutical ingredients. The new resolution addresses the requirements for assessing the risk to human health of veterinary medicinal products, and methods of analysis for the purpose of conformity assessment. In the case of active pharmaceutical ingredients with antimicrobial properties, the risk assessment includes resistance parameters associated with an adverse impact on human health. ANVISA Normative Instruction no. 51/2019 establishes, among others, the list of maximum residue limits (MRLs), acceptable daily intake (IDA) and acute reference dose (DRFA) for 138 active pharmaceutical ingredients of veterinary medicines in foods of animal origin.

The first programme on antimicrobial use in food animals was introduced in 2005 and focussed on the control of residues in food products. MAPA is responsible for carrying out the National Plan for the Control of Residues and Contaminants (PNCRC), which sets out the approaches to monitoring and controlling residues in all foods of animal origin. The results of the PNCRC are regularly published. In 2014, the first official initiative to monitor AMR was undertaken by the Ministry of Fisheries and Aquaculture. However, in 2015 this monitoring role was transferred to MAPA. In 2017, MAPA noted that any changes to antimicrobial use in animal production are based on scientific evidence and risk analysis, and priority is given to the use of the critically important antimicrobials $(\mathrm{CIA})$ in human health.

In October 2017, the Normative Instruction 41 was published, and established the National Programme for the Prevention and Control of Antimicrobial Resistance in Agriculture (AgroPrevine). This programme attempts to strengthen measures for the prevention and control of antimicrobial resistance in livestock, within the One Health framework. The co-ordination of this Programme is under the responsibility of the Department of Animal Health of MAPA. In essence, this programme aims to promote activities related to the objectives and strategic interventions established in the National Action Plan for the Prevention and Control of Antimicrobial Resistance (PAN-BR AGRO), which was launched on 16 May 2018 and covers the period 2018-2022. These activities include health education, epidemiological studies, surveillance and monitoring of antimicrobial use, strengthening of measures to prevent AMR infections and controls, and the promotion of the responsible use of antimicrobials in animal production.

The sectoral plan on tackling antimicrobial resistance in animal agriculture (PAN-BR AGRO) has been merged with the National Plan on antimicrobial resistance in human health under the PAN-BR. The National Plan targets five strategic objectives and is aligned to the Global Action Plan (WHO/FAO/OIE). More specifically, the National Plan is composed of eight broad objectives, 15 strategic interventions and 21 activities. These are discussed in the following sections.

\section{National Action Plan on Antimicrobial Resistance within the Scope of Agriculture and Livestock (PAN-BR AGRO) 2018-2022}

The main strategies of the Plan are to:

- To improve awareness and understanding of antimicrobial resistance through education, training and effective communications.

- To strengthen knowledge through surveillance and research.

- To reduce the incidence of infections through effective prevention measures.

- To optimize the use of antimicrobials in livestock.

- To examine the economics of investments in alternative medicines and vaccines and other interventions for implementation of the Plan and ensure sustainable development.

To achieve these objectives the detailed interventions and actions are also described in the Plan. In an effort to raise public awareness of AMR in agriculture and the food sector, a national communications plan has been developed and includes stakeholders in animal production, animal health and the food industry. In addition, there is increased emphasis on the supervision of antimicrobials in animal production, as well as the proper disposal of unused drugs. 
A further key activity is the emphasis on improving the understanding and capacity of stakeholders along the agri-food supply chain of AMR, especially farmers and veterinarians. In this context, the Plan highlights the need to harmonise the guidelines on the training of farmers and farm workers in tackling AMR across the different States.

The establishment of an integrated surveillance and monitoring system for AMR across the livestock sector is seen as a key to obtaining better data and information on antibiotic use and resistance. This system has been introduced for broiler chicken production, and is expected to be extended to other food animal species at a future date. Another aspect of the monitoring of antibiotic use in animal production involves regular quality checks on veterinary antibiotics used in production. Further research into chemical and non-chemical alternatives to antibiotics in animal production is also part of this activity.

The emphasis on sanitary and biosecurity practices in livestock production is a high priority activity in order to enhance and control the emergence and spread of infectious diseases in animals. The adoption of good agricultural practices is a central plank to optimizing the use of antimicrobials in animal production. This activity is linked through the national regulations on the rational use of antimicrobials in food animal production.

A further aspect of the National Action Plan is the setting of an annual budget for the implementation of the various activities as part of the Federal Budget Planning. For agriculture, the specific budget is included in the MAPA Annual Operational Plan. In addition, alternative funding sources are also explored in order to implement the Plan and tackle the rise in AMR. These sources include public/private funding initiatives and partnerships with international organisations.

\section{Summary}

The Health Surveillance Agency (ANVISA) is responsible for monitoring the use of antibiotics and antimicrobial resistance in humans and in food. The Ministry of Agriculture, Livestock and Food Supply (MAPA) is responsible for monitoring the use of antibiotics and antimicrobial resistance in animals and in food. Although still at an early stage of implementation, the National Action Plan has been successful in raising public attention to the use of antibiotics in animal production and in bringing about changes in the veterinary medicine legislation on antibiotics. In Brazil, antibiotics have been widely used in animal production to prevent and treat bacterial diseases, and to promote animal growth. The consumption of antibiotics is predominantly in the intensive pig and poultry sectors, while cattle production is largely based on extensive grazing systems. In recent years, the volume of antimicrobials consumed in pigs and poultry production has been declining in response to the significant changes in the legislation on antimicrobials, and the withdrawal of specific antibiotics from the recommended list of approved antibiotics for use in animals. In addition, consumer driven concerns in both the export and domestic markets have also contributed to this decline.

While official figures on changes in antibiotic use in livestock production are not available, several studies have suggested a substantial reduction in antibiotic use in the poultry and pig sectors. In recent years, Brazil has aligned its policies and standards with those recommended by International Organisations such as the OIE and Codex Alimentarius. As a result, Brazil introduced substantial changes in its legislation on the use of antibiotics in animal production, including banning antibiotics in animal feed for growth promotion, as well as limiting the use of antibiotics for prophylactic purposes.

For pig and poultry producers, in particular, one of the major challenges has been the rise in production costs due to the stricter limits on the range of antimicrobials that can be used in production. Concerns over the potential loss of competitiveness has resulted in livestock producers searching for effective and low cost alternatives to maintain animal health and productivity. Livestock producers are starting to adopt alternative methods to prevent and control animal diseases including increased investment in better husbandry practices, biosecurity and hygiene measures, combined with the selective use of vaccinations at different stage of the animal's lifecycle.

Changes in production practices such as improving feed and water quality and lowering the stocking density, are likely to take time and may require further investments by producers in these sectors. Nevertheless, the implementation of the core measures set out in the National Action Plan such as raising public awareness of AMR and increased training, investment in alternative interventions, and improved co-ordination amongst 
stakeholders is progressing, and research into identifying effective alternative chemical and non-chemical interventions to antibiotics in animal production is on-going.

\section{China}

China is the largest global producer of veterinary antimicrobials, driven by the demand from its large and growing animal production sector. With the key policy priority on food security, antibiotics are widely used in animal feed, for the treatment of animal diseases, as well as for promoting growth on livestock farms. With the increasing intensification of animal production, and the relatively weak hygiene conditions on many farms, the use of antimicrobials was increasing until 2016. However, the implementation of the National Action Plan to Contain AMR (2016-2020), and substantial changes in the regulations for the sale and use of antibiotics in animals have contributed to a sharp decline in antibiotic usage in recent years. In addition, the outbreak of African Swine Fever (ASF) in 2019 has resulted in an estimated decline of about $40 \%$ in the swine herd, and this has resulted in a sharp fall in demand for veterinary antimicrobials in pig production.

In China, the use of antimicrobials in animal production is an important part of the food safety system. Antimicrobial use is regulated under the Chinese Food Safety Law, which came into force in 2009 and has been adjusted several times to improve food safety in recent years. Most of the antimicrobials used in animal production are administered through feed additives. In overall terms, pig and poultry production account for over $80 \%$ of antimicrobial use (62\% and $23 \%$, respectively), driven by the rapid growth in the number of intensive production farms in these two sectors.

While there are no official figures published on the overall use of antibiotics in animal production, several researchers have estimated the usage at between 84-97 000 tonnes (Wu 2018). While most of these antimicrobials are specifically produced for use in veterinary medicine, a substantial quantity of antibiotics for human medicine are also used in animal production. However, since the implementation of the NAP, the use of human antimicrobials in animal production has declined. In addition, changes in the regulations on the classes of antimicrobial permitted for animal use, and improvements in the quality of veterinary antibiotics have contributed to these changes.

In August 2016, the Chinese Government released the National Action Plan to Contain Antimicrobial Resistance. This Plan covered the five-year period (2016-2020), and sets out the detailed strategies, targets and actions to tackle AMR. The Plan adopts the One Health approach to tackling AMR and includes the five critical pillars as set out in the GAP. More specifically, the Plan outlines specific targets on antibiotic use, the development of new antimicrobials, the sales of antibiotic only through prescriptions, optimization of surveillance, the rational use of antibiotics in animals and in humans and the provision of information to the public on AMR controls. The Plan adopts a multi-sectoral approach to tackling AMR and is co-ordinated by the Chinese National Health and Family Planning Commission (NHFPC), in conjunction with 14 other ministries. The Ministry of Finance also participates and provides the funding for the implementation of the Plan. The Ministry of Agriculture and Rural Affairs (former Ministry of Agriculture) is responsible for the implementation of the Action Plan in animal production.

\section{National Action Plan to Contain AMR 2016-2020}

The National Action Plan includes measures across the three sectors; human, animal and environmental health, to combat the risks of AMR. The Plan enabled a framework to be established which promoted better co-ordination across the different sectors and stakeholders. An important component of this framework focused on securing sustainable investment in research and development and strengthening the regulatory system for the production, sale and importation of antimicrobials. A further feature of the Plan focused on education and capacity building for veterinarians and farmers in order to optimize the use of antimicrobials and to mitigate the emergence of AMR. More specifically, the central goals of the Plan are to:

- Enhance public awareness about antimicrobials and promote the education and training programmes for stakeholders.

- Ensure that all antibiotics for human and animal medicine are by prescription.

- Improve the surveillance system on antibiotics use and AMR in both humans and animals.

- Enhance the programme for antimicrobial stewardship. 
- Launch 1-2 new antimicrobial drugs and develop 5-10 diagnostic techniques.

- Phase out the use of antibiotics as animal growth promoters.

China's NAP goes beyond the five pillars as set out in the WHO's Global Action Plan. The goals are more comprehensive and specific and cover a range of other issues including, the prevention of environmental pollution, as well as the development of new antibiotics and therapeutic drugs. Given the complexities inherent in controlling drug resistance across China, the implementation of the NAP faced many challenges, in particular, the co-ordination of responses across Ministries, livestock sectors and regions.

The NAP proposed the following targets, the launch of one to two new antimicrobial drugs, and the development of five to ten new diagnostic techniques. In addition, the surveillance and monitoring of antimicrobial use and antimicrobial resistance in human health and in animal production was a high priority. Another feature of the Plan was to improve the management and control of the circulation and sale of antibiotics for veterinary medicine. Moreover, the NAP also emphasises the importance of basic research, new technologies and the development of infrastructure as central to the implementation of the Plan and tackling AMR in China.

A further key aspect of the NAP was the raising of public awareness of AMR and the potential consequences to human and animal health, and food security. The importance of increasing human capacity and understanding of AMR through education and training programmes for stakeholders as a critical activity in the implementation process. The NAP also advocates greater co-operation with international organisations and assistance for other countries in the region in containing the risks of antimicrobial resistance.

The implementation of the prescription only system for antibiotic use in animal production together with the enforcement of the stricter regulations have contributed to reducing the overuse of antibiotics in the animal sector. The NAP outlined specific targets and responsibilities for all sectors, as well as the reward and punishment system for departments and individuals who fail to implement the provisions of the Plan. With its very diverse animal production systems, China has faced many administrative and technical barriers to adopting new animal farming practices that reduce the need for the use of antibiotics in production. The ultimate success of the NAP will likely depend on how these barriers are addressed across the different sectors.

\section{Summary}

On the intensive, high-density pig and poultry farms, antibiotics are often seen as a prophylactic (insurance) measure to prevent the outbreak of an infectious disease. Moreover, the poor sanitation conditions on many animal farms have also contributed to the demand for antibiotics in the feed. However, an important factor driving the inappropriate use of antibiotics in animal production is their low cost relative to the perceived benefits in enhancing animal growth and feed efficiency. Several studies indicate that the use of antibiotics for animal growth promotion account for about two-thirds of the total antibiotics used in food producing animals.

In recent years, changes in the legislation on antimicrobial use in animal production have resulted in a decline in the use of antibiotics in animal feed. In 2016, the Ministry of Agriculture phased out the use of eight veterinary drugs in food animal production. In addition, the enforcement of the regulations on the withdrawal periods prior to animal slaughter, and the mandatory requirement of prescriptions for all antimicrobials used for both human and animal health has improved.

An important aspect of the NAP, in addition to outlining the technical targets and policies, has been the setting of financial guarantees to ensure its implementation. While additional financial support is allocated to improve biosecurity measures, the NAP also outlines the responsibility of the Ministry of Finance to allocate sustainable resources for the implementation of all activities over the five-year period of the Plan.

With its comprehensive scope and ambitious targets, the implementation of the involved in the implementation of the Plan is complex across such a diverse range of activities and targets. Moreover, with the One Health approach to tackling AMR, it is important that these measures are implemented consistently across all sectors. While the overall co-ordination mandate is the responsibility of the NHFPC (National Health and Family Planning Commission), the co-operation between departments in different Ministries can 
be problematic. In addition, the adoption of better preventative measures often requires farmers' to change their management practices, in particular, to improve farm hygiene and sanitary standards.

In China, AMR has resulted in a high incidence of multidrug resistant bacteria (Wu, 2017). However, with greater efforts to increase public awareness, the education and training of stakeholders including, veterinarians and farmers, have contributed to lowering the inappropriate use of antibiotics in animal production.

Enforcement of the regulations on antimicrobial use in animal production, and the phasing out of certain medically important antibiotics, are critical to the successful implementation of the NAP. Overall, estimates indicate that China has used about five times more antimicrobials (mg/PCU) in pig and poultry production than the international average (Wu 2018). Some research studies suggest that antibiotics can be substantially over-used (by as much as $90 \%$ ) in poultry production. However, while there has been progress in reducing the inappropriate use of antimicrobials, there is urgent need to improve the regulations on the disposal of antibiotics and animal waste to control the rise of AMR in the environment.

A dilemma for Chinese policy makers is how to balance the national food security concerns with the health risks to humans and animals from the emergence and spread of AMR. There have been several significant developments in 2020 in relation to the implementation of the NAP in animal production. First, the Government announced that the production of AGPs would cease, as of January 2020, and from July 2020, all AGPs were banned for use in food producing animals. Second, the implementation of the AMU Reduction Plan in food animals (2018-21) resulted in 100 trial farms being selected to undertake experiments on lowering the use of antibiotics for different animal species in different regions. The results reported from the majority of the trial farms showed that the phasing out of AGPs was possible without having any significant impact on animal productivity. Lastly, research into developing new antimicrobial drugs, as alternatives to antibiotics, from plant extracts is being explored in the Chinese Academy of Agricultural Sciences.

\section{Russian Federation}

In September 2017, the government of Russia approved the "The Strategy for Preventing the Spread of Antimicrobial Resistance in the Russian Federation up to 2030". The Ministry of Health together with other Federal Ministries have outlined a series of measures for implementing the Strategy. The Strategy defines the state policy to prevent and limit the emergence and spread of resistance to antimicrobials, as well as chemical and biological agents in the Russian Federation. In Russia, the use of antibiotics in agriculture is regulated for all food products.

In 2019, the 2019-2024 National Action Plan for the implementation of the Strategy for preventing the spread of antimicrobial resistance in Russia was approved. The Plan includes provisions for improving state regulations on the use of antimicrobial drugs in healthcare and veterinary medicine, as well as the development and updating of clinical recommendations on the provision of medical care for infectious and parasitic diseases, as well as prevention of infectious diseases. In addition, the Plan also includes the organisation and monitoring of residual antibiotics in food raw materials and food products, conducting information campaigns on antimicrobial resistance and increasing the level of professional training of specialists. Also the Plan prohibits the use of antibacterial drugs in productive animals for prophylactic purposes. Medical supplies must undergo the state registration procedure, within which indications for the use of each drug in certain doses and a scheme of use are clearly established in order to prevent the use of antibacterial drugs for prophylactic purposes or as growth promoters. Currently, only ionophores, flavophospholipol and bacitracin are used for prophylactic purposes.

The Ministry of Agriculture regulates the use of antibiotics in animal production, while the Ministry of Health regulates the use of antimicrobials in healthcare and is responsible for intersectional coordination of the implementation of the AMR Strategy as a whole. The professional community of clinical microbiologists has developed a comprehensive map of antimicrobial resistance in relation to human health (online platform for analysis, visualization and exchange of data on antibiotic resistance_AMR cloud), and submitted it to the Ministry of Health. The Russian State Centre for Quality and Standardization of Veterinary Drugs and Feed (VGNKI), under Rosselkhoznadzor, is the Federal State Institution conducts both theoretical and applied research aimed at ensuring quality of veterinary drugs and safety of feed, products of animal and plant origin. It is also engaged in certification of veterinary drugs, feed and feed additives. Within the framework of the One Health approach VGNKI together with the Research Institute of Antimicrobial Chemoterapy subordinate 
to the Ministry of Health, carry out similar work in the field of veterinary and animal husbandry. An open project was launched on the AMR cloud platform with the results of the research work "Veterinary monitoring and risk analysis of antibiotic resistance of zoonotic bacteria".

Rosseklhoznadzor and the Russian Federal Service for Surveillance on Consumer Rights Protection and Human Wellbeing (Rospotrebnadzor) play an important role in monitoring the level of antibiotics residues in food products and antibiotic resistant bacteria. The Surveillance Centre works closely with international organisations in developing its monitoring system. The Reference Center --Rospotrebnadzor was created in 2017 on the basis of the Federal Budgetary Institution of Science "Central Research Institute of Epidemiology" of Rospotrebnadzor. The Reference Centre and Rosselkhoznadzor monitor the residual amount of antibiotics and antibiotic resistance of bacteria in food raw materials and food. Main functions of Reference Center are: detecting of the residual amount of antibiotics in food raw materials and food products; detecting of contamination of food raw materials and food products with microorganisms with the determination of AMR of the isolated strains; providing advisory and methodological assistance to territorial bodies and organizations of Rospotrebnadzor, organizations and institutions of the EAEU and CIS countries, including according to FAO programs. Our Reference center is now undergoing procedures to obtain the status of the FAO Reference Center for Antibiotic Resistance for our region. In addition, Russia is a member of the G20 Global Research and Development Center for AMR (Global R\&D Hub).

Policies to tackle antimicrobial resistance have a high priority in Russia and approaches to deal with AMR are set out in the "Fundamentals of State Policy in the Field of Chemical and Biological Safety of the Russian Federation for the period to 2025 ".

\section{Strategy for preventing the spread of antimicrobial resistance in Russia for the period up to 2030}

On 25 September 2017, the Russia signed a decree "On adoption of the Strategy for Preventing the Spread of Antimicrobial Resistance in the Russian Federation for the period to 2030". This strategy is based on the One Health approach and includes nine core chapters to combat the rise in AMR. On 30 March 2019, the Action Plan (2019-2024) for the implementation of the strategy was approved. More specifically, these are to:

- Improve public awareness on the use of antimicrobials (AMU) and antimicrobial resistance (AMR).

- Increase the quality of specialists' training in relevant industries on issues related to AMR.

- Improve measures to prevent and limit the spread of pathogens with antimicrobial resistance.

- Enhance the systematic monitoring of AMR. Develop universal MIC - plates with antimicrobials to determine the AMR of isolates from food for strengthening the surveillance systems that monitoring antimicrobial use and AMR

- Develop new antimicrobial drugs and alternative methods including technologies for the prevention, diagnosis and treatment of infectious diseases in humans, animals and plants.

- Intensify the development and introduction into agricultural practice of a national guidelines of vaccinations to prevent zoonoses and reduce the consumption of antimicrobial drugs in veterinary medicine.

- Improve measures and regulations to control the spread of antimicrobials and biological agents, as well as prohibition on the use of antibiotics for prophylactic purposes

- Increase and develop coordination among the human, animal, plant and environmental health sectors in order to develop and promote the concept of "One Health" through the creation of a single intersectoral committee.

- Enhance intersectoral co-operation and international co-operation to prevent and limit the spread of antimicrobial resistance. 
The Action Plan emphasises the need for better public communications on the threats from AMR and the need educational programmes on the appropriate use of antimicrobials and alternative treatments for infectious diseases for specialists. In addition, the Plan focuses on the implementation of the national antimicrobial regulations, including better control over the prescription of antimicrobials for human medicine and for veterinary purposes. Closer monitoring of the use of antimicrobials for disease prevention and a prohibition of all advertising of antimicrobials, as well as stricter controls on the use of antimicrobials in animal production and in feed additives are foreseen.

A further key emphasis is on enhancing professional standards and qualifications for specialist responsible for monitoring antimicrobial use. The Action Plan focuses on new effective methods to diagnose the profile of drug resistant pathogens and the implementation of sanitary-epidemiological and veterinary supervision. In addition, the Action plan calls for the development and adoption of alternative methods, technologies and biological drugs to antibiotics in treating animal and plant diseases. Another core aspect relates to measures on the traceability of antimicrobials that are manufactured in Russia and those that are imported into Russia.

The strategy for preventing the spread of antimicrobial resistance is being implemented in two stages. The first stage focuses on raising public awareness and the rational use of antibiotics in animal production and the training of specialists on AMR. The second stage involves the implementation of the remaining measures outlined in the strategic Plan.

\section{Summary}

The main result of the implementation of the Strategy in Russia is ensuring application of strict control on the use of antibacterial agents for prophylaxis and growth promotion. The introduction of strict control over the procedure for prescribing antibacterial drugs (prescription dispensing) will allow to limit use of antimicrobial drugs and more accurately assess the effectiveness of their use.

Regulatory authorities are developing programmes for the rational use of antibacterial agents in veterinary based on the recommendations of the OIE, taking into account drugs that are critical for human medicine.

The Federal Service for Veterinary and Phytosanitary Surveillance (Rosselkhoznadzor) and Reference Centre Rospotrebnadzor carry out epidemiological monitoring of antibiotic residues and AMR bacteria in raw materials and food, as well studies and analysis of phenol-and genotypic signs of antibiotic-resistant isolates if microorganisms in Russia and countries of Eurasian region; collects data on AMR bacteria and antibiotic residues detected in raw food materials or food products.

\section{Lessons learned and best practices to contain the risks of AMR}

Many countries had measures in place to tackle the risks posed by AMR in human health and in animal health long before the release of the Global Action Plan (WHO 2015). However, the release of the GAP not only helped to draw greater awareness to the challenges posed by AMR, but set out a more coherent and cross sectoral approach to tackling AMR through the One Health framework. Most countries have drawn on this broad framework. Subsequently, many countries developed their country specific national action plans on AMR, covering human, animal, plant, and environmental health. While many countries are still at an early stage in implementing their national action plans (NAP), this preliminary assessment identifies lessons that can be drawn such as changes in husbandry practices (e.g. improved nutrition, lower housing density, onfarm biosecurity measures, selective use of vaccines), to reduce the risks of AMR in animal agriculture. The adoption of the One Health approach to tackling AMR has resulted in improved transparency and the adoption of more systematic and coherent policies across the different sectors.

With the extensive global awareness campaigns on the threats posed by AMR, stakeholders are better informed and have a better understanding of the potential risks to human, animal and environmental health posed by the emergence and spread of antimicrobial resistance. The overarching goal of the NAPs is to reduce risks to human and animal health, and the environment, posed by the rise in bacterial resistance to antibiotics. At the national level, this approach brings important benefits, particularly by encouraging different sectors to work together and share their experiences and practices in a more consistent and transparent way. In addition, there are likely to be important long-term benefits such as reducing the incidence of other 
infectious animal diseases from this more inclusive approach to combatting AMR. Moreover, there is evidence of spill-over effects of AMR between sectors and the transmission of resistant genes and bacteria between species that may require sustainable solutions for animal and human health.

The use of antimicrobials in animal production in OECD countries, although decreasing, varies widely between countries and across different animal sectors. In many countries, the pig and broiler chicken sectors account for the largest share of antibiotic use in food producing animals due to their high stocking density and intensive nature of production. Thus, measures that influence the use of antibiotics in these two sectors can have a substantial impact on the overall use of antibiotics in food producing animals, and consequently, the selection pressure on microbes to develop resistance.

This study assesses the changes in food animal production practices, in particular, the use of antibiotics in production in the six OECD countries (Australia, Canada, Denmark, Ireland, Japan, and the United States), as well as in Brazil, China and Russia. These countries differ in many aspects, including the type of animal production system, national regulations on antibiotics, and their administrative structures to address AMR. Overall, there has been a significant fall in the use of antibiotics in animal production, with the largest decreases recorded in those countries that have implemented stricter regulations on antibiotics use together with better surveillance programmes and data collection. Contributing to this decline has been the phasing out of antibiotics for animal growth promotion. Moreover, the evidence also suggests that more and more countries are limiting the use of the highest priority critically important antibiotics for animal use, and limiting the use of antibiotics, such as fluoroquinolones, third and fourth generation cephalosporins and colistin for animal use, as well as the use of antibiotics as a prophylactic in livestock systems. However, in some countries, there is increasing concern on the shift to more potent antibiotics, and these changes can be hidden in the overall quantity figures on antibiotic use in animal production.

This assessment shows that intervention efforts to tackle AMR should be tailored by country, farming type, and food animal species. In many countries, policies are focussed on antimicrobial stewardship programmes at the country and sector level. Moreover, the availability of antibiotics only through veterinary prescriptions has enabled better stewardship of existing antibiotics and has helped to reduce the inappropriate use of antibiotics in maintaining animal health and welfare. In addition, many countries have undertaken rigorous analysis of the risks associated with AMR in food producing animals and this has underpinned their specific regulations, and has enabled these countries to make significant progress in tackling resistance in the different food animal species.

With the extensive national and international awareness programmes on the risks posed by the rise in AMR, good progress has been made in raising public understanding of the issue, and in bringing needed changes to the traditional animal production systems. In some countries, this has resulted in changes in consumer preference for animal products. More and more, consumers are becoming better informed about the products they consume, and the types of medicines used in the production system. In response to these changes, farmers have been seeking alternative products to antibiotics in their animal production systems. More specifically:

- Changes in the market demand for meat and meat products are changing the economic incentives facing livestock producers, and to focus on products that are produced with prudent (or controlled) use of antibiotics.

- This has resulted in greater interest in alternative interventions in production that are technically and economically feasible at the farm level.

This assessment shows that most countries have adopted a mix of different policies and measures to tackling AMR ranging from the top-down measures to the bottom-up measures, to influence the behaviour and production practices of livestock producers. This study also shows that no single measure or solution can address the challenges posed by AMR. Rather, a mix of policy measures and good husbandry practices can successfully and sustainably reduce the need for antibiotics in livestock production, and consequently lower the risks of AMR. These findings show that progress has been made, in tackling the threats from AMR, but that more needs to be done especially in relation to understanding the different animal production systems. The following sections highlight some of the lessons learned and outstanding policy challenges to combatting the rise in AMR in animal production. 


\section{Policies to prevent disease outbreaks are central to addressing the risks of AMR}

The assessment suggests that the central focus of a mitigation strategy should be based on preventative measures that limit and prevent infectious disease outbreaks in food animals. More specifically, the findings highlight the crucial importance of good farm management, sound veterinary advice, and good animal husbandry practices to ensure good animal health, welfare, and high animal productivity. Together, these actions not only change the need for antibiotics on animal farms, but also bring additional positive externalities in terms of reducing the incidence of other non-bacterial animal diseases. These practical measures tend to be mutually supportive and sustainable across the different food animal systems.

For livestock farmers, the adoption of good biosecurity measures both on the farm and off the farm is critical. These measures include stricter controls over the movement of animals, transport vehicles and farm workers, greater attention to hygiene on the farm, ensuring correct isolation procedures for sick animals, and the isolation of all incoming animals to the farm.

Improving animal husbandry practices on livestock farm also plays an important role. For example, closer attention to animal housing conditions, in particular, having appropriate ventilation and stocking density in animals sheds. In addition, improving the nutrition of animals also strengthens the animals' natural resistance to disease, and greater attention to the breeding of animals that may carry higher resistance to specific bacterial diseases.

Lastly, the selective use of vaccines and professional veterinary advice to prevent specific bacterial infections has proved to be effective in some situations and some production systems. In practice, however, vaccinations are usually done as a package of measures including the adoption of good management and biosecurity measures in animal production.

\section{Good regulations that encourage the responsible use of antibiotics in food animal production}

While regulations on the use of antibiotics in animal production differ significantly between countries, the enforcement of robust and transparent regulations is an important policy tool in tackling AMR. In some countries, a lack of harmonisation of the regulations on the sale and use of antibiotics for food animals can limit the effectiveness of these regulations, but good co-ordination between the different levels of government is critical. In many countries, the requirement for veterinary prescriptions for antibiotics in animal production has made a significant contribution to a more efficient use of antibiotics in production. In addition, government regulations have an important role to play in restricting veterinary counterfeit drugs and assuring the quality of the antimicrobials that are available on the market, and in limiting access to non-prescription (over-thecounter) antimicrobials for agricultural use, particularly in low to middle income countries.

Effective antimicrobial regulations emphasise the role of risk analysis and risk management in developing appropriate policies on antibiotic use in food animals. While most countries undertake a rigorous approach to the analysis of risks associated with AMR, countries often differ in their interpretation of the assessment of the estimated risks involved. However, the lack of data on the actual use of the different classes of antibiotics by animal species often limits the risk analysis exercise. While the enforcement of regulations is challenging in many countries, an increasing emphasis on the role of antibiotic stewardship has contributed to limiting the inappropriate use of antibiotics in animal production.

At the international level, the ad hoc Codex Intergovernmental Task Force on Antimicrobial Resistance (TFAMR) is revising the Codex Alimentarius Code of Practice to Minimize and Contain Antimicrobial Resistance. This document sets out a series of indicators and incentives for the responsible use of antimicrobials in order to minimize the development of resistance in pathogens.

Lastly, a transparent and pragmatic regulatory system based on input from all stakeholders, including relevant non-government entities is important in developing and implementing regulations, and the adoption of alternative interventions to antibiotics in animal production (e.g. probiotics, bacteriophages). These alternative products are likely to contribute to reducing the risks of antimicrobial resistance in the future, however, further research is still needed in this area. For some countries (relatively high users of antimicrobials), there is likely to be a lack of transparency or willingness to divulge the actual usage of antimicrobials for fear of having some negative impacts on trade and market access. 


\section{Integrated and reliable surveillance and monitoring systems of antimicrobial use and antimicrobial resistance}

A comparative analysis of best practices and mitigation policies across sectors and countries requires having an internationally standardised approach to collecting and reporting information on antibiotic use and antibiotic resistance in animals. Many countries have adopted an approach developed by the OIE Terrestrial and Aquatic Animal Health Codes. In addition, countries are at different stages of advancement in collecting data on antibiotic use in animal production and much of the reporting is based on sales data, which is a crude indicator of actual antibiotic use. However, several countries have started to collect farm level data on antimicrobial use by species, and this will facilitate better targeting of AMU policies in the future. Having a reliable surveillance system is central to ensuring the appropriate use of antibiotics in animal production and to the targeting of policies on AMR. The Codex Alimentarius (TFAMR) is also elaborating the Guidelines for the Integrated Monitoring and Surveillance of Foodborne Antimicrobial Resistance in response to the needs of Member countries as they implement their NAPs and, in particular, their integrated approach to foodborne AMR risk management.

The data collected by the national surveillance systems have allowed some countries to establish "benchmarking" for the use of antibiotics in the different animal sectors. The setting of benchmarks and specific targets can improve the effectiveness of specific classes of antibiotics in food producing animals. In addition, this allow some comparison between the different animal sectors in the country, as well as with similar sectors in other countries.

In the livestock sector, reliable and accurate data on the use of antibiotics by different food animal species and stage of production is not yet available in any country. However, many countries, as part of their NAPs, have started to collect granular data on antimicrobial use and antimicrobial resistance in animal production. These data, once available, may provide valuable input to policy makers and improve the targeting of policies aimed at tackling AMR.

Lastly, countries that have an efficient and integrated monitoring system are better able to identify where antibiotic waste occurs, and where there are difficulties in the disposal of unused antibiotics. They can then take the appropriate actions to resolve these problems. At the farm level, the benefits also include the identification of high antibiotic users, which allows the direct and targeted guidance to help those farmers optimise their antibiotic usage.

\section{Well-functioning and coherent administrative structures to include all stakeholders}

In many countries, a major challenge is how to address the issue of transparency and coherence in the implementation of policies to combat AMR in humans, animals and the environment. The sharing of experiences across different administrative structures, relevant non-government organisations and institutions in different sectors can be challenging due to the priority given to AMR and the nature of the organisation. The co-ordination of AMR policies between different levels of government, different ministries, regulators, veterinarians and farmers is complex and can often lead to misunderstandings and slow implementation of the policies needed to tackle AMR. However, the One Health approach, which has been adopted by most countries, helps to overcome some of these challenges as it facilitates a more coherent implementation of AMR policies across the different sectors.

Lastly, a number of countries have established high-level taskforces to oversee the implementation of the NAPs, and this has contributed to improving implementation, as well as improved collaboration amongst stakeholders. In addition, this approach has facilitated an assessment of the effectiveness of the policies on a regular basis, as well as addressing problems that may arise within or between sectors. Finally, improving the co-operation between public policy institutions and the private stakeholders in tackling AMR is critical to developing long-term sustainable solutions to this enormous challenge.

\section{Having adequate funding to implement the national action plans}

The availability of adequate resources is critical to implementing the policies as set out in the NAPs to mitigate the impacts of antimicrobial resistance in animal agriculture. In many countries, the NAPs do not 
deal with the identification and allocation of funding to tackle AMR, and this lack of funding has slowed the implementation of measures to combat resistance across the sectors. In some countries, most of the public funding is allocated to dealing with AMR in human health, and, there is little funding left to implement policies on AMR in animal, plant and environmental health.

This report does not address the issue of who should bear the cost of antimicrobial resistance mitigation (e.g. producers, consumers, taxpayers), but this issue can be part of some future work on AMR. Some countries have developed new initiatives to deal with the lack of funding for AMR, and these initiatives often involve different types of collaboration and partnerships between the public and private sectors. While still at a relatively early stage, these initiatives may offer a potential solution to overcoming the lack of resources to implement measures to combat AMR across all sectors.

The resources (funds, expertise and infrastructure) required to implement the measures outlined in the national action plans can be significant. Finding adequate funding for public awareness programmes, training and education, developing the surveillance and monitoring systems, as well as acquiring the expertise to implement the policies and to check compliance with regulations, is a major challenge for most countries.

\section{Outstanding challenges and shortcomings of the national action plans}

In many countries, good progress has been made in implementing the various actions and measures specified in the National Action Plans. Nevertheless, there are several outstanding challenges that need to be addressed, and these include the following:

- Under the One Health approach for tackling AMR, the overarching goal has been on developing coherent policies across the three pillars; human health, animal health and the shared environment. However, the impacts of resistance bacteria in the environment of importance to human medicine are not well known. In many of the NAPs, there is limited focus on the actual environmental impacts of resistance bacteria and the necessary funding to address these concerns. The lack of focus on the environment is often due to a sparsity of information and data in this area. However, a tighter targeting of the NAPs to specific aspects of the environment, where actions could have a high impact on containing the risks of AMR to human and animal health.

- Greater efforts to understand the transmission of antimicrobial resistance between different animal species, and between humans, animals and the environment are needed. A better understanding of the modes of transmission and the key drivers of this transmission is critical in order that countries can develop effective and sustainable strategies to combatting AMR. Improving integrated AMR surveillance is an essential part of this process.

- Countries have made good progress in improving their regulatory systems on antimicrobials destined for human and animal health; however, the lack of proper enforcement of these regulations is a cause for concern in many countries.

- The lack of economic information on the mitigation costs of AMR in the different animal production systems continues to be an obstacle to effectively tackling this problem. Further research on estimating the cost effectiveness of alternative interventions in animal production would be an important step to identifying sustainable alternative products and practices to antibiotics in production, and, thus to reducing the dependence on antibiotics.

- Any strategy on tackling AMR will require clarity in the amount and duration of the budget allocations to implement the various mitigation measures. In addition, greater efforts could be made to develop public- private partnerships to ensure that adequate funding is made available to successfully implement the mitigation measures needed to contain the risks of AMR.

- The sharing of experiences, knowledge and solutions across sectors, countries and regions is important in order to maximise the return on investments in AMR mitigation measures. This could be achieved, for example, through improved co-ordination via international organisations. 


\section{References}

Aarestrup, F. M., Jensen, V. F., Embourg, H. D., Jacobsen, E., and H.C. Wegener (2010). "Changes in the use of antimicrobials and the effects on productivity of swine farms in Denmark". American Journal of Veterinary Research , 2010, 71(7)726-733.

Asai, T., Hiki, M., Ozawa, M., Koike, R., Eguchi, K., Kawanishi, M., Kojima, A., Endo, Y.S., Hamanoto, S., and Tatsuro Sekiya (2014). "Control of the Development and Prevalence of Antimicrobiakl Resistance in Bacteria of Food Animal origin in Japan: A new Approach for Risk Management of Antimicrobial Veterinary Medicinal Products in Japan". Foodborne Pathogens and Disease, Volume 11, No 3, 2014. Japan.

Australian Department of Health and the Department of Agriculture (2015). "Responding to the Threat of Antimicrobial Resistance". Australia's First National Antimicrobial Resistance Strategy 2015-2019. Canberra, Australia.

Australia's National Antimicrobial Strategy - 2020 and Beyond (2020). Australian Government, Department of Health, Department of Agriculture, Water and the Environment, Canberra, Australia.

Australian Pesticides and Veterinary Medicines Authority (2014). "Quantity of Antimicrobial Products sold for Veterinary Use in Australia, July 2005 to June 2010". Canberra, Australia.

Bondt, N. and H. Kortstee. "Good Practices: Use of Antibiotics". 2016 L.E.I., Wageningen UR,

Netherlands." https://www.government.nl/documents/reports/2016/01/27/good-practices-use-ofantibiotics

Bokma, M., Bondt, N., Neijenhuis, F., Mevius, D., and Stephanie Ruiter. 2014. "Antibiotic use in Brazilian broiler and pig production: an indication and forecast of trends. Report No 714. Wageningen UR Livestock Research, LEI, Netherlands.

Brazil's Ministry of Agriculture, Livestock and Food supply (2018). National Action Plan For the Prevention and Control of Antimicrobial Resistance within the Scope of Agriculture and Livestock (PAN-BR AGR 2018-2022). Ministry of Agriculture, Livestock and Food Supply. Brasilia, Brazil.

Cardinal, K.M. et al. " Simulação doimpacto produtivo da retirada de antibióticos promotores de crescimento da ração de frangos de corte' . In: Conferencia FACTA-WPSA Brasil. Campinas May 16 th to $17^{\text {th }} 2018$.

Cecchini M, et al. (2015). "Antimicrobial resistance in G7 countries and beyond: Economic Issues, Policies and Options for Action". Paris: OECD Publishing, 2015.

Codex Alimentarius (2011). "Guidleines for Risk Analysis of Foodborne Antimicrobial Resistance", Rome, Italy.

Collineau, L., et al. (2017) "Herd-specific interventions to reduce antimicrobial usage in pig production without jeopardising technical and economic performance". Preventive Veterinary Medicine 144 (2017) 167-178.

Cordoso, M., (2019), "Antimicrobial use, resistance and economic benefits and costs to livestock producers in Brazil". OECD Food, Agriculture and Fisheries Paper №132, OECD, Paris.

Cui $D$, et al. "Use of and microbial resistance to antibiotics in China: A path to reducing antimicrobial resistance". Journal of International Medical Research. 2017:0300060516686230.

Department of Health (2017). Ireland's National Action Plan on Antimicrobial Resistance 2017-2020 (iNAP). Dublin. Ireland. http://health.gov.ie/national-patient-safety-office/patient-safetysurveillance/antimicrobial-resistance-amr/.

DTU Food \& SSI (2015). DANMAP 2014. Use of Antimicrobial Agents and Occurrence of Antimicrobial Resistance in Bacteria from food animals, Food and Humans in Denmark. (http//www.danmap.org).

DVFA (2018). The Yellow Card Initiative on Antibiotics. Glostrup, Denmark. 
European Food Safety Authority (2016). The European Union Summary report on Antimicrobial Resistance in Zoonotic and Indicator Bacteria from Humans, animals and Food in 2014. Available at: https:// www.efsa.europa.eu/en/efsajournal/pub/4380

European Commission (2017). A European One Health Action Plan against Antimicrobial Resistance (AMR). Available at: https://ec.europa.eu/health/amr/action_eu_en.

European Medicines Agency (2016). Sales of Veterinary Antimicrobial Agents in 29 European Countries in 2014. Available at: https:// www.ema.europa.eu/docs/en_GB/document_library/Report/2016/10/WC500214217.pdf

European Medicines Agency (2017). Sales of Veterinary Antimicrobial Agents in 30 European Countries in 2015. Seventh European Surveillance of Veterinary Antimicrobials Consumption (ESVAC) Report. London. U.K.

FAO (2011). One Health: Food and Agriculture Organization of the United Nations Strategic Action Plan. Rome, Italy.

FAO (2016). "The FAO Action Plan on Antimicrobial Resistance 2016-2020". FAO, Rome, Italy.

FAO (2018). "Antimicrobial Resistance, Policy Review and Development Framework". FAO, Bangkok, Thailand.

FAO, Ministry of Environment and Food of Denmark (2018). Tackling Antimicrobial Use and Resistance in production: Lessons Learned in Denmark, Rome, Italy.

Food Safety Authority of Ireland (2015). Potential for Transmission of Antimicrobial Resistance in the Food Chain. Available at: https://www.fsai.ie/publications_AMR.

Government of Japan (2016). "National Action Plan on Antimicrobial Resistance 2016-2020". Tokyo, Japan.

Government of Canada (2017). "Tackling Antimicrobial Resistance and Antimicrobial Use: A PanCanadian Framework for Action". Ottawa, Ontario, Canada.

Hennessy, D.A., J. Roosen and H.H. Jensen (2005), "Infectious disease, productivity, and scale in open and closed animal production systems", American Journal of Agricultural Economics, Vol.87, pp. 900-917.

Hoelzer, K., Wong, N., Thomas, J., Talkington, K., Jungman, E. and A. Coukell (2017). "Antimicrobial drug use in food-producing animals and associated human health risks: what, and how strong, is the evidence?". BMC Veterinary Research (2017) 13:211. The Pew Charitable Trusts, Washington, DC 20004.

$\mathrm{Hu} \mathrm{Y}$, Cheng $\mathrm{H}$. "Use of veterinary antimicrobials in China and efforts to improve their rational use". Journal of Global Antimicrobial Resistance. 2015;3(2):144-6.

Hosoi, Y., Asai, T., Koike, R., Tsuyuki, M., and K. Sugiura (2014). "Sales of Veterinary Antimicrobial Agents for Therapeutic Use in Food producing Animal Species in Japan between 2005 and 2010". Rev. Science Tech. Off. Int. Epiz., 2014, 33(3) 1007-1015. Tokyo, Japan.

LandersT.F., Cohen, B., Wittum, T.E., Larson, E.L. (2012). "A review of Antibiotic Use in Food animals: Perspective, Policy and Potential”. Public Health Reports, 2012; 127 pp 4-22.

Laxminarayan, R., Matsoso, P., Plant, S., Brower, C., Rottingen, J.A., Klugman, K., Davies, S. (2015). "Access to Effective Antimicrobials: A Worldwide Challenge". Lancet 387: 168-75.

Ministry of Agriculture. Bureau of Veterinary, Ministry of Agriculture PRC. 2009中国动物卫生状况报告 (Animal Health in China): 中国农业出版社; 2011.

Morel, C., (2019). "Transmission of antimicrobial resistance between animal agriculture and humans", OECD Food, Agriculture and Fisheries Paper N¹34, OECD, Paris, France, 2019.

Ministry of Environment and Food \& Ministry of Health of Denmark (2017). One Health Strategy against Antibiotic Resistance. Copenhagen, Denmark. 
OECD (2018). "Stemming the Superbug Tide: Just A Few Dollars More". OECD Health Policy Studies. OECD.

OIE (2020). Annual Report on the Use of Antimicrobial Agents Intended for Use in Animals: OIE 2020. https.//www.oie.int/fileadmin/Home/eng/Our_scientific_expertise/docs/pdf/Fourth_Annual_Report_A MU.pdf.

OIE (2016). Report of the 2nd International Symposium on Alternatives to Antibiotics. OIE, Paris, France.

OIE (2018). List of Antimicrobial Agents of Veterinary Importance. OIE, Paris, France. https://www.oie.int/fileadmin/Home/eng/Our_scientific_expertise/docs/pdf/AMR/A_OIE_List_Antimicr obials_May 2018.pdf.

OECD (2018). Stemming the Superbug Tide: Just a few Dollars More, OECD Health Policy Studies, OECD Publishing, Paris, https://ideas.respec.org/b/ohe/monogr/000464.html.

OECD (2020). "Tackling Antimicrobial Resistance from a One Health Perspective: Progress on model enhancement to assess effectiveness and cost-effectiveness of policies for antimicrobial resistance prevention and control in OECD and G20 countries". Paper presented to the Expert Group on the Economics of Public Health on 13 October 2020. OECD, Paris.

OIE (2017). Annual Report on the Use of Antimicrobial Agents in Animals: Better Understanding of the Global Situation. OIE, Paris, France.

OIE (2016). World Organisation for Animal Health. "The OIE Strategy on Antimicrobial Resistance and the Prudent Use of Antimicrobials". OIE, Paris, France.

OIE (2015). "OIE Standards, Guidelines and Resolution on Antimicrobial Resistance and the Use of Antimicrobial Agents". OIE, Paris, France.

Postma, M., Backhans, A., Collineau, I., Loesken, S.,Sjolund, M., Belloc, C., Emanuelson, U.,Grosse Beilage, E., Stark, K.D., and J. Dewulf (2016). The Biosecurity Status and its Associations with Production and Management Characteristics in Farrow to Finish pig Herds. Porcine Health Management 2:9 DOI 10.1186/s40813-016-0028-z. Ghent University, Belgium.

Public Health Agency of Canada (2017). "Pan-Canadian Framework for Action on Antimicrobial Resistance and Antimicrobial Use". Ottawa, Ontario, Canada.

Public Health Agency of Canada (2018). "Canadian Antimicrobial Resistance Surveillance System 2018". Ottawa, Ontario, Canada

Rushton,J., Coyne,L., Patrick,I., Williams, N., Carrique-Mas, J.,Dinh, B.T., and D. Moran. "The use of Economics for Selecting Interventions and Policies". Centre of Excellence for Sustainable Food Systems. N8 Research Partnership: Agrifood. University of Liverpool, U.K.

Rushton, J. (2013). "An Overview Analysis of Costs and benefits of Government Control Policy Options". In Proceedings of Livestock Disease Policies: Building Bridges between Science and Economics. International Workshop held in OECD, Paris, France, 3-4 June 2013, Pages 39-51.

Rushton, J., J. Pinto Ferreira and K.D. Stark (2014), "Antimicrobial Resistance: The Use of Antimicrobials in the Livestock Sector", OECD Food, Agriculture and Fisheries Papers No 68, OECD Publishing.

Rushton, J. (2015). "Antimicrobial use in animals: How to Assess the Trade-Offs". Zoonoses and Public Health 62, 10-21.

Ryan, M. (2019). Evaluating the Economic Benefits and Costs of Antimicrobial use in Food producing Animals. OECD, Food, Agriculture and Fisheries Paper No 132. OECD Publishing. Paris. France.

Singer, R.S., Porter, L. (2019). Estimates of On-Farm Antimicrobial Usage in Broiler Chicken and Turkey Production in the United States, 2013-2017. Mindwalk Consulting Group, LLC, 2019. Falcon Heights, MN, USA.

Teillant, S., Laxminarayan, R. (2015). "Economics of Antibiotic Use in US Swine and Poultry Production". 
The Magazine of Food, Farm and Resource Issues. 1st Quarter, 2015.

UNEP(2017), "Antimicrobial Resistance: Investigating the Environmental Dimension". UNEP, Nairobi. Kenya.

The AMR One Health Surveillance Committee (2017). "Nippon AMR One Health Report (NAOR) 2017". Tokyo, Japan.

US Food and Drug Administration, Center of Veterinary Medicine (2018). The 2017 Summary

Report on Antimicrobials Sold or Distributed for Use in Food Producing Animals. Washington, D.C., US Avaialble at: https://www.fda.gov/media/119332/download.

U.S White House (2015). The National Action Plan for Combating Antibiotic Resistant Bacteria 20152020. Washington. United States.

US White House (2014). The National Strategy for Combating Antibiotic Resistant Bacteria 2015-2020.

World Bank (2016). "Drug-Resistant Infections: A Threat to Our Economic Future. Part VI. Antimicrobial Use in Animals and AMR, pp 65-78", Washington, DC. Washington. United States.

US Executive Office of the President, President's Council of Advisors on Science and Technology (2014). Report to the President on Combating Antibiotic Resistance. Washington, United States.

WHO (2015). "Global Action Plan on Antimicrobial Resistance".World Health Organization, Geneva, Switzerland, 2015.

(Available

at: http//wpro.who.int/entity/drug_resistant/resources/global_action_plan_eng.pdf).

WHO (2017). "WHO Guidelines on Use of Medically Important Antimicrobials in Food-Producing Animals".World Health Organization, Geneva, Switzerland.

WHO. World Health Organization. 2017. Highest Priority Critically Important Antimicrobials. Available at: http://www.who.int/foodsafety/cia/en/ Accessed on September $9^{\text {th }} 2018$.

WHO (2015). "Antimicrobial Resistance: Global Report on Surveillance", Geneva. Switzerland.

WHO (2003). "Impacts of antimicrobial growth promoter termination in Denmark". The WHO international review panel's evaluation of the termination of the use of antimicrobial growth promoters in Denmark. Geneva, Switzerland.

Wu Z. (2017). "Balancing food security and AMR: a review of economic literature on antimicrobial use in food animal production". China Agri Econ Rev. 2017;9(1):14-3

Wu, Z., (2019). "Antibiotic use and antibiotic resistance in food producing animals in China", OECD Food, Agriculture and Fisheries Paper №133, OECD, Paris, France, 2019.

Wu Z. (2018), "Antimicrobial use in food animal production: Situation analysis and contributing factors", Frontiers of Agricultural Science and Engineering, Vol. 5(3), pp. 301-311.

Wu Z. Balancing food security and AMR: a review of economic literature on antimicrobial use in food animal production. China Agri Econ Rev. 2017;9(1):14-31.

Xiao Y. (2017). "A National Action Plan to Contain Antimicrobial Resistance in China: Contents, Actions and Expectations". AMR Control. 2017. 


\section{OECD FOOD, AGRICULTURE AND FISHERIES PAPERS}

The report was declassified by the OECD Working Party on Agricultural Policies and Markets in November 2020 and was prepared for publication by the OECD Secretariat.

This report, as well as any data and any map included herein, are without prejudice to the status of or sovereignty over any territory, to the delimitation of international frontiers and boundaries and to the name of any territory, city or area.

The statistical data for Israel are supplied by and under the responsibility of the relevant Israeli authorities. The use of such data by the OECD is without prejudice to the status of the Golan Heights, East Jerusalem and Israeli settlements in the West Bank under the terms of international law.

Comments are welcome and can be sent to tad.contact@oecd.org.

(C) OECD (2021)

The use of this work, whether digital or print, is governed by the Terms and Conditions to be found at http://www.oecd.org/termsandconditions. 